\title{
Collusion through Communication in Auctions
}

\author{
Marina Agranov* Leeat Yariv ${ }^{\dagger \ddagger}$
}

October 26, 2015

\begin{abstract}
We study the extent to which communication can serve as a collusion device in one-shot first- and second-price sealed-bid auctions. Theoretically, second-price auctions are more fragile to collusion through communication than first-price auctions. In an array of laboratory experiments we vary the amount of interactions (communication and/or transfers without commitment) available to bidders. We find that the auctioneer's revenues decrease significantly when bidders can communicate. When, in addition, bidders can make transfer promises, revenues decline substantially, with $70 \%$ of our experimental auctions culminating in the object being sold for approximately the minimal price. Furthermore, the effects of communication and transfers are similar across auction formats.
\end{abstract}

Keywords: Auctions, Communication, Collusion, Experiments

${ }^{*}$ Division of the Humanities and Social Sciences, Caltech. E-mail: magranov@hss.caltech.edu ${ }^{\dagger}$ Division of the Humanities and Social Sciences, Caltech. E-mail: lyariv@hss.caltech.edu

${ }^{\ddagger}$ We thank Dino Gerardi, Ben Gillen, Sotiris Georganas, Stephen Morris, and Jean-Laurent Rosenthal for useful discussions and suggestions and Joy Basinger, Tatiana Mayskaya and Li Song for superb research assistance. We gratefully acknowledge financial support from the National Science Foundation (SES 0963583) and the Gordon and Betty Moore Foundation (Grant 1158). 


\section{Introduction}

\subsection{Overview}

Collusion has been a long-standing problem for auction design. Krishna (2002) reported that in the 1980s, collusion and auctions went hand-in-hand: $75 \%$ of U.S. cartel cases involving collusion were auction-based. To date, approximately $30 \%$ of antitrust cases filed by the Department of Justice since 1994 involved bid-rigging in industries such as construction, antique sales, military supplies, utility procurement, etc. ${ }^{1}$ The prevalence of collusion in auctions has led to a substantial body of theoretical work in the Economics literature. By and large, this literature has taken two approaches to explaining the emergence of collusion: through repeated interactions between bidders, and through bidding that occurs over multiple objects (simultaneously or over time). Roughly speaking, both approaches allow bidders to devise joint schemes in which winning bidders alternate over time or over objects. Winning prices are low, because at each period, or for each object, only a select group of bidders is bidding competitively (see our literature review below). We suggest an alternative, possibly complementary, channel through which collusive outcomes may emerge. Namely, we study the effects of communication in one-shot, sealed-bid, first- and second-price auctions. We provide theoretical and experimental results that suggest that communication alone can affect auction outcomes dramatically.

As a motivating example, consider a sealed-bid second-price auction with two bidders, with independent private values drawn from $\{0,1\}$ with equal probabilities for each value. The unique equilibrium in weakly undominated strategies entails both bidders bidding their values. This would suggest a price of 0 whenever at least one of the bidders values the object at 0 , and a price of 1 when both bidders value the object at 1 . Suppose now that bidders participate in a pre-play communication phase after learning their values, but prior to bidding, and consider the following protocol. Bidders reveal their values. If either has a value of 0 , they both bid their values. However, if both have a value of 1 , they toss a fair coin. If it falls on Heads, bidder 1 submits a bid of 0 and bidder 2 submits a bid of 1 . If the coin falls on Tails, bidder 1 submits a bid of 1 and bidder 2 submits a bid of 0 . This profile constitutes part of an equilibrium: bidders have no incentive to lie nor to submit bids different than those prescribed. Furthermore, the price of the object under such a protocol is always 0 . Communication allows bidders to coordinate their bids, contingent on their values.

In our theoretical analysis (Section 3) we consider sealed-bid first- and second-price auctions with two bidders whose values are independently and privately drawn from a uniform

\footnotetext{
${ }^{1}$ According to the authors' tabulation, 438 cases out of 1423 antitrust cases involved bid-rigging, see: http://www.justice.gov/atr/cases/
} 
distribution. In such settings, collusive outcomes, particularly ones corresponding to very low revenues, are associated with a reduction in efficiency. We show that, as in the example, the set of equilibrium outcomes expands when cheap-talk communication is available in second-price auctions and a revenue of 0 is possible even when bidders use a symmetric strategy profile. ${ }^{2}$ In contrast, in first-price auctions, low prices cannot be achieved uniformly in equilibrium, even when communication is available. In fact, we demonstrate that the set of outcomes generated by second-price auctions with communication strictly contains those generated by first-price auctions. In theory, then, second-price auctions are potentially more fragile to collusion through communication than first-price auctions.

Analyzing the impacts of communication on real-world auction outcomes is difficult to do. Communication is often tacit and unobserved, and private values, available information, etc. are unknown. Laboratory experiments are therefore particularly useful as initial tests of the impacts of communication in auction settings.

We report results from an array of experimental first- and second-price auctions with two bidders. For each auction format, we run three treatments. The first corresponds to auctions without communication, mimicking some of the original designs of experimental auctions (e.g., Cox, Roberson, and Smith, 1982, Dyer, Kagel, and Levin, 1989, Kagel and Levin, 1993, and Harstad, 1991). In our second treatment, subjects freely communicate using an instant messaging screen after observing their private values and prior to bidding. Our third treatment is a modification of the second treatment - subjects freely communicate after observing their values and before bidding and, furthermore, can transfer money to one another (in one simultaneous decision) after seeing the auction results, i.e. the winning bidder and the object's price. Importantly, in all our sessions we randomly match bidders in each round and in several sessions we employ a complete-strangers protocol in which subjects are never rematched with the same individual. This assures that there are no repeated interaction effects in our design and any collusive behavior is an artifact of the one-shot interaction alone.

The results from our treatments without communication replicate those already noted in the literature. Bidders over-bid in both auction formats and efficiency levels (the probabilities with which the high-value bidder wins the object) are high, hovering around $80 \%$.

Communication by itself leads to significant price drops, reducing the auctioneer's revenues by up to $33 \%$. From a theoretical perspective, the availability of ex-post transfers should have no effect on outcomes (since there is no incentive to transfer money after the

\footnotetext{
${ }^{2}$ In the example above, the bidders effectively randomize between the symmetric equilibrium, and two asymmetric equilibria (in which one bidder bids 0 and the other 1). In Section 3 we show that the set of equilibrium outcomes strictly contains the convex hull of the equilibrium outcomes without communication.
} 
fact). However, the availability of transfers makes a substantial difference in the lab. In particular, the reduction of prices is especially stark in sessions in which both communication and transfers are available. In fact, in about $70 \%$ of both first- and second-price auctions in which communication and transfers were available, the object's price is zero. As a consequence, the availability of communication and transfers significantly reduces revenues under both auction formats, yielding less than one third of the revenues generated when all communication is banned under either auction format. This is interesting in view of the Department of Justice data - transfers were explicitly mentioned in 148 of the reported 438 antitrust cases involving bid-rigging.

Nonetheless, a sort of Revenue Equivalence Theorem result holds in our data as revenues are not significantly different from one another across auction formats, in all our treatments. Furthermore, the availability of communication and/or transfers does not significantly affect efficiency levels.

When inspecting the communication protocols, we see that subjects tend to discuss two types of strategies. One, which we term flip-a-coin, entails subjects both submitting the same (often low) bid and thereby generating a fifty-fifty chance of each receiving the object; The other, which we term reveal-collude, entails subjects revealing their values and then submitting bids that assure a low price and the high-value bidder winning the object. The flip-a-coin strategy is simpler for subjects to manipulate; One of the bidders need only submit a slightly higher bid than agreed to win the object. Indeed, while that strategy is discussed at fairly high rates, particularly when only communication is available, it is infrequently followed. In contrast, the reveal-collude strategy is often followed when discussed and is the predominant strategy subjects discuss when transfers are available.

In terms of what subjects reveal during communication, the availability of transfers makes a substantial difference. Without transfers, subjects often do not share their private values or their intended bids. When they do, they often understate both their values and the bids they plan to submit. When transfers are available, misrepresentation diminishes significantly and truthful revelation of values and bids is part of the modal communication protocol.

We also see a persistent pattern of behavior at the transfers stage. When the ultimate price is high, indicating the bidders did not successfully collude, transfers are rare. However, when ultimate prices are close to zero, winning bidders submit an average of $44 \%$ of their surplus, with a modal transfer of $50 \% .^{3}$ This suggests a coherent description of how subjects achieve collusive outcomes: they first share their values and then bid in a way that allows the

\footnotetext{
${ }^{3}$ Positive transfers would not be predicted to occur in any sequential equilibrium of the corresponding games. Indeed, in the transfer stage, in principle, there is no reason to pass on a positive amount. Violations as such occur in many other types of games, such as the dictator game, the trust games, the centipede game, etc. (see Kagel and Roth, 1997).
} 
high-value bidder to win the object at a low price (in the first-price auction, this means that both bidders submit a low bid, in the second-price auction, at least the losing bidder submits a low bid). The winning bidder then shares her surplus with the losing bidder, so long as the final price is low. This behavior is in line with several case studies of bid rigging. For instance, Pesendorfer (2000) studied bidding for school milk contracts in Florida and Texas during the 1980s. His data suggest that in Florida, the school milk cartel used side payments to compensate bidders for refraining from bidding competitively, which is effectively what we see in our experimental data.

The behavior we observe also hints at why collusive outcomes are easier to achieve when transfers are available. Without transfers, losing bidders, who must submit low bids to generate a collusive outcome, do not gain from their behavior. The only beneficiary is the winning bidder. In contrast, transfers allow subjects to align their preferences.

Since much of the literature on collusion in auctions has focused on repeated interactions (see our literature review below), we also ran an auxiliary set of experiments in which subjects participated in a sequence of repeated auctions, with or without communication. When auctions are repeated, a substantial difference between first- and second-price auctions emerges. Repeated first-price auctions yield greater revenues than repeated second-price auctions, with or without communication. Importantly, for either auction format, repetition with or without communication does not lead to a greater scope for collusion than communication and transfers do in one-shot interactions. This observation is particularly pronounced for first-price auctions, in which repetition and communication together generate significantly higher prices than those produced in the one-shot variant with communication and transfers.

To summarize, our results indicate the substantial scope for collusion that communication and transfers allow, even in one-shot settings. Together, they lead to the minimal feasible

revenue with a substantial probability under both auction formats. Repetition, one of the common explanations for the emergence of collusion, is not more effective, even when agents can communicate. The persistent patterns of behavior seen in our data are not in line with some of the basic theoretical predictions, however, and suggest the need for a richer theoretical framework.

\subsection{Related Literature}

The empirical literature documents many cases in which bidders in a variety of auction formats colluded (see, for instance, Hendricks and Porter, 1989 and Marshall and Marx, 2012 for reviews).

Following the prevalence of bid-rigging in auctions, a large body of theoretical work 
on collusion in auctions has emerged. Much of this work analyzes settings with repeated interactions, in which, roughly speaking, bidders can collude by devising schemes that split the auctions won over time (see Abreu, Pearce, and Stachetti, 1986, Athey and Bagwell, 2001, and Skryzspacz and Hopenhayn, 2004). Another approach considers multi-object auctions, in which collusive outcomes can emerge from bidders "splitting the market"; namely, bidders decide on which objects whom will bid on competitively (see, e.g., Kwasnica, 2002 and Brosco and Lopomo, 2002). Several papers study how communication affects the set of equilibrium outcomes in sealed bid one-shot auctions. McAfee and McMillan (1992) show that cartels can achieve full efficiency in auctions with side transfers and pre-stage communication and commitment. Without transfers, the best payoffs the cartel can achieve are generated by either non-cooperative bidding or bid rotation schemes, in which the winner is decided upon independently of her value. Without commitment, Lopomo, Marx, and Sun (2011) show that in an independent-value setting, a bidding ring operating at a first-price sealed-bid auction cannot achieve any gains relative to non-cooperative bidding, a result we will see is special to first-price auctions. ${ }^{4}$

In terms of experimental work, our paper relates to the strand of experimental literature that studies behavior in one-shot sealed-bid auctions (see Kagel and Levin, 1993, Cox, Roberson, and Smith, 1992, and the surveys by Kagel, 1995 and Kagel and Levin, 2011). However, to our knowledge, the question of how cheap-talk communication affects behavior in one-shot sealed-bid auctions has not been tackled before - that is our main contribution. ${ }^{5}$

Llorente-Saguer and Zultan (2014) study collusion in laboratory auctions by testing the model of Eso and Schummer (2004). They allow one bidder, whose identity is determined prior to valuations being realized, to "bribe" the other. A bidder who accepts a bribe is committed to stay out of the auction. Bribes then serve as a signaling instrument. In their setting, outcomes under first- and second-price auctions are also similar. In addition, failed collusion attempts decrease efficiency, particularly in first-price auctions. This design connects with some of our auxiliary sessions allowing for commitment in our setting, which we discuss in Section 11.3.

Outside the realm of auctions, a growing experimental literature explores the effects of

\footnotetext{
${ }^{4}$ Matthews and Postlewaite (1989) study $k$-double auctions with a bidder and a seller who have private values of a good. Communication enlarges the set of equilibrium outcomes and renders the set of equilibrium outcomes identical across all $k$-double auctions, $k \in[0,1]$. Bergemann, Brooks, and Morris (2013) study twobidder first-price auctions with private values and identify the information structures that minimize revenues, which in turn link to the information structures buyers may desire collectively to increase their surplus.

${ }^{5}$ Isaac and Walker (1995) study the effects of face-to-face communication in a repeated first-price, private value, sealed-bid auction. They restricted the communication protocols by banning discussion of private values or side payments. Kagel (1995) studied collusion in first-price common value auctions with reserve prices, under similar restrictions on communication protocols. There is also some experimental work focusing on repeated auctions and multi-object auctions (see Section 4.1 in Kagel and Levin, 2011).
} 
cheap-talk communication on strategic outcomes; see Crawford (1998) for a survey of some early literature, most of which placed strict restrictions on the messages sent. Recently, more studies have focused on free-form unrestricted communication rather than structured restricted communication. Unrestricted communication has been shown to affect strategic behavior of subjects in various environments, including hidden-action games (such as trust games) and hidden-information games as in Charness and Dufwenberg (2006, 2011), weak link games as in Brandts and Cooper (2007), bargaining as in Agranov and Tergiman (2014) and Baranski and Kagel (2015), collective action settings as in Goeree and Yariv (2011), and public good games as in Oprea, Charness, and Friedman (2015). One coherent message emerging from this body of work is that communication promotes coordination on Pareto superior outcomes. In all these settings, however, the outcomes observed with communication tend to benefit all participating individuals. In contrast, in the auction environment, a collusive outcome in which the object is won at a low price entails a huge asymmetry between players: only one bidder (the winner) benefits from driving the final prices down, while others do not gain from colluding. In particular, it is hard to extrapolate existing results to the auction environment.

\section{Experimental Design}

We use a sequence of first-price and second-price independent private-value auctions involving two bidders. ${ }^{6}$ In all of our experimental auctions, each bidder bids for one object, the value of which is drawn independently from a uniform distribution over $[0,100]$, where each experimental point corresponds to 1 cent. In all of our treatments, both bidders submit a bid. The winner of the object is the highest bidder, generating the value of the object to the winner (and no reward for the loser of the auction). The price, paid only by the winner of the object, is given by the highest bid in the first-price auction and the lowest bid in the second-price auction; ties are broken randomly. Sessions varied in the amount and type of interaction that was available to bidders.

No Communication. In these treatments, subjects observe their private values and then asked to simultaneously submit their bids. These treatments replicate the classical experimental auctions a-la, e.g., Kagel and Levin (1993).

Pure Communication. In these treatments, subjects observe their private values and are then able to communicate via an instant messenger screen. When at least one bidder decides

\footnotetext{
${ }^{6}$ The full instructions are available at: http://www.hss.caltech.edu/ ${ }^{\sim}$ lyariv/papers/Collusion_Instructions.pdf
} 
to stop communication (expressed by a clickable button on the experimental interface), both bidders are asked to simultaneously submit their bids.

Communication with Transfers. In these treatments, subjects observe their private values, are then able to communicate freely, and, after communication comes to a halt, submit bids simultaneously (as in the pure communication treatment). Once bids are entered, the results of the auction are observed by both bidders. Then, each bidder can choose to make a transfer to the other bidder (greater or equal to zero). The ultimate payoff for each bidder is their auction payment plus their net transfers.

To summarize, the experiments employ a $2 \times 3$ design based on the variation in the auction format (first-price and second-price) and the type of interaction available between bidders. Each experimental session implemented one combination of auction format and interaction type. Three to six sessions were run for each treatment. ${ }^{7}$ Most sessions entailed one practice round followed by 10 periods of actual play, and subjects were randomly paired in each period. ${ }^{8}$ In several of the sessions, we employed a complete strangers protocol, in which subjects were never paired more than once with another subject. While such sessions require more subjects, they allow us to eliminate repeated game effects altogether.

In addition, in several sessions we elicited risk attitudes using the Gneezy and Potters (1997) methodology. ${ }^{9}$ Namely, at the end of each of these sessions, we asked subjects to allocate 100 points (translating into $\$ 2$ ) between a safe investment, which had a unit return (i.e., returning point for point), and a risky investment, which with probability $50 \%$ returned 2.5 points for each point invested and with probability $50 \%$ produced no returns for the investment. Any amount earned from this task was added to the overall earnings in the session. Table 1 summarizes our design details.

The experiments were conducted at the Experimental Social Sciences Laboratory (ESSL) at University of California at Irvine. Overall, 296 subjects participated. The average payoff per subject was $\$ 22$, including a $\$ 12$ show-up fee.

There are a few points to note regarding our design choices. First, we allowed subjects to communicate freely rather than offer them a restricted set of messages, which would have arguably made the analysis simpler. Our decision was due to several reasons: while laboratory auctions certainly have artificial features that do not perfectly match real auctions, we did want to make the communication as organic as possible. In fact, the endogenous

\footnotetext{
${ }^{7}$ For one treatment, an additional session was run, but due to some slow subjects, the number of rounds was low. This session produced indistinguishable results from our other sessions. However, since for much of our analysis we focus on rounds 6 and on, we excluded this session from our data.

${ }^{8}$ In several sessions we allowed for subjects to go through up to 15 rounds. However, due to time constraints, most of the sessions were run with 10 rounds only.

${ }^{9}$ This method is among the more popular ones to elicit risk attitudes of subjects in laboratory experiments (see survey of Charness, Gneezy, and Imas, 2013).
} 
Table 1: Experimental Design

\begin{tabular}{llcc}
\hline \hline Auction Format & Available Interaction & Num of Subjects & Rounds* $^{*}$ \\
First-price & No Communication & 30 & $(10,10,10)$ \\
& Pure Communication & 72 & $\left(15,15,15, \mathbf{1 0}^{* *}, \mathbf{1 0}^{* *}, \mathbf{1 0}^{* *}\right)$ \\
& Communication with Transfers & 48 & $\left(10,11,12, \mathbf{1 0}^{* *}\right)$ \\
Second-price & No Communication & 36 & $(10,10,10)$ \\
& Pure Communication & 64 & $\left(10,10, \mathbf{1 5}^{* *}, \mathbf{1 0}^{* *}, \mathbf{1 0}^{* *}\right)$ \\
& Communication with Transfers & 48 & $\left(10,10,10, \mathbf{1 0}^{* *}\right)$ \\
& & & \\
\hline \hline
\end{tabular}

Remarks: * Each entry corresponds to the number of rounds run in a session. ${ }^{* *}$ Sessions run with a complete strangers protocol. Numbers in bold correspond to sessions in which risk was elicited.

communication protocols were something we wanted to inspect. As will be seen, different treatments led to different communication protocols that would have been hard for us to predict (and thereby design an appropriate set of restricted messages). In addition, we were concerned that by restricting communication protocols we would guide subjects toward particular patterns of behavior, thereby introducing a form of experimenter demand.

Second, we study auctions with only two bidders. Many auctions entail a fairly small number of bidders. For instance, the eBay analytics team reported to us that in 2013, 27\% of auctions with multiple bidders had only two bidders participating (and $77 \%$ had five or fewer bidders). ${ }^{10}$ We believe the analysis of two-bidder auctions is a natural first step to studying collusion through communication: they are simple in that any negotiation is only between two individuals and communication is a two-way interaction. We view the study of auctions with more bidders as an interesting direction for the future.

Third, our design of the treatment allowing for transfers takes a very special form. In particular, we did not allow subjects more sophisticated contractual mechanisms, namely ones that would allow them to commit to, possibly contingent, transfers. In a way, the transfer instruments we provide are fairly weak (theoretically, they should have no impact at all), and yet, as we will see, they have a dramatic impact on outcomes. In that respect, we suspect that more elaborate transfer instruments may enable even greater levels of collusion. In Section 11.3 we return to this point and report results from auxiliary sessions that allowed for a particular form of commitment to transfers.

Last, we study sealed-bid first- and second-price auctions as opposed to English and Dutch auctions. Sealed-bid auctions are prevalent in applications and are simpler to implement in

\footnotetext{
${ }^{10}$ Hong and Shum (2002) also report a small number of bidders in general highway, construction, and grading and paving procurement auctions and Grether, Porter, and Shum (2014) report a small number of bidders (with averages ranging between $2-5$ ) in used automobile auctions.
} 
the lab with communication since there is a natural point in time for communication to occur. Studying the effects of communication on English and Dutch auctions, as well as other auction formats, is also left for the future.

\section{Theoretical Background}

The basic results we present in this section are tailored to our experimental design. We assume there are two risk-neutral bidders. Agents' private valuations are taken from $V=$ $[0,100]$ according to distribution $F$. That is, at the outset, each individual knows her own value realization, but not the other bidder's. Bids are restricted to $B=V=[0,100]$.

We concentrate on first- and second-price auctions in which the highest bidder receives the good for a price corresponding to the highest and second highest bid, respectively. In either auction format, upon a tie, the winner is randomly chosen.

In our setting, absent communication, the first-price auction admits a unique equilibrium in which each bidder submits half her valuation (see Lebrun, 2004 and Maskin and Riley, 2003). In the second-price auction, there is a unique symmetric equilibrium, which entails strategies that are not weakly dominated, where each bidder bids precisely her value (see Fudenberg and Tirole, 1991). Nonetheless, there exist multiple asymmetric equilibria (for instance, one bidder bidding 100 and the other 0, regardless of their private values, is an equilibrium). ${ }^{11}$ It is important to note that when symmetric equilibria are played in the firstand second-price auctions, the resulting mappings between bidders' valuations to allocation of the object (i.e., the probability that either bid wins the object) coincide. Furthermore, in both auctions the bidder with a valuation of 0 expects 0 payoffs. In this case, the Revenue Equivalence Theorem applies and the first- and second-price auctions are expected to generate identical revenues, given by $100 / 3$.

In what follows, we consider the case, corresponding to some of our treatments, in which a cheap-talk stage is available after agents learn their private valuations and prior to submitting their bids. Formally, we consider the cheap-talk extensions of the first- and second-price auctions and study the induced set of equilibria.

From the Revelation Principle, an equilibrium with communication is tantamount to a mapping $\mu: V^{2} \rightarrow \Delta\left(B^{2}\right)$ satisfying two types of incentive constraints: truthful revelation and obedience. An equilibrium outcome is a mapping $\gamma: V^{2} \rightarrow \Delta(\{1,2\} \times[0,100])$, which maps any reported value profile into a distribution over the winning bidder and the price paid for the object. Denote by $\Gamma_{k}$ the set of equilibrium outcomes corresponding to the

\footnotetext{
${ }^{11}$ For three or more bidders, Blume and Heidhues (2004) characterize the full set of equilibria in secondprice auctions without communication.
} 
$k$-price auction with communication, $k=1,2$.

Notice that in any equilibrium with communication of either the first- or second-price auction, an agent with a valuation of 0 expects a payoff of 0 . From the Revenue Equivalence Theorem, it follows that all efficient equilibria, ones allocating the object to the highestvaluation bidder, generate the same revenue (calculated, as before, to be 100/3). Therefore, a reduction in prices following communication must go hand-in-hand with a reduction in efficiency and we have the following observation:

Observation 1 (Collusion and Efficiency): Any equilibrium of either the first- or secondprice auction with communication that generates expected prices lower than $100 / 3$ is inefficient: the highest-value bidder does not always receive the object.

We now turn to the scope of collusion. Notice that in a second-price auction with communication, bidders can guarantee a price of 0 , even in a symmetric equilibrium. Namely, bidders can randomly select the winner of the auction, who then submits a bid of 100, while the other bidder submits a bid of 0 . This cannot be replicated in the first-price auction, where a price of 0 requires both bidders to submit a bid of 0 and is subject to profitable deviations. In fact, in the first-price auction, there cannot be any equilibrium of the game with communication in which the price of the object is always below some $p$, for sufficiently small $p$. Intuitively, suppose in some equilibrium the price always falls below some $p>0$. A bidder who submits a bid of $p+\varepsilon$ for an arbitrarily small $\varepsilon>0$ would then win the object for sure. For a bidder with valuation $v>p$ to follow the equilibrium prescriptions instead requires that the bidder win the object with a sufficiently high probability and, in that case, pay a price lower than $p+\varepsilon$. For instance, if $v-p>v / 2$, or $v>2 p$, a bidder with a valuation of $v$ would need to win the object with more than a $50 \%$ probability. But, since $v$ is distributed uniformly on $[0,100]$, if $p<25$, more than half of the bidders have a probability greater than $50 \%$ of winning the object, which leads to a contradiction. ${ }^{12}$ To summarize, we have the following observation:

Observation 2 (Full Collusion under First- and Second-price Auctions): In the firstprice auction, there is no equilibrium with communication that generates a price lower than $100 / 4=25$ regardless of the realized valuations. In the second-price auction, there is a (symmetric) equilibrium with communication that yields a price of 0 always.

\footnotetext{
${ }^{12}$ Lopomo, Marx, and Sun (2011) showed that when there are two bidders and two possible values for the object, if the bid increment is sufficiently small, profitable collusion is not possible. While the generalization of this result to our setup is beyond the scope of this paper, these observations are in line with their conclusions.
} 
Observation 2 suggests that full collusion, generating 0 revenue, is possible under the second-price auction, but not under the first-price auction. The next proposition illustrates that, in fact, any outcome produced in the first-price auction with communication can be emulated in the second-price auction with communication. In that respect, with communication, the scope for collusion under the second-price auction is strictly greater than that under the first-price auction. Formally,

Proposition 1: The set of equilibria outcomes generated by second-price auctions with communication strictly contains all equilibrium outcomes generated by the first-price auction, $\Gamma_{1} \subsetneq \Gamma_{2}$.

Proof: Notice that the outcomes corresponding to equilibria of the second-price auction without communication (and any mixtures of those) remain equilibrium outcomes of the second-price auction with communication. We now show that the outcome corresponding to the unique equilibrium in the first-price auction can be emulated in the second-price auction with communication. Indeed, consider the following mapping:

$$
\mu\left(v_{1}, v_{2}\right)= \begin{cases}\left(100, \frac{v_{1}}{2}\right) & v_{1}>v_{2} \\ \left(\frac{v_{2}}{2}, 100\right) & v_{1}<v_{2} \\ \frac{1}{2} \otimes\left(100, \frac{v_{1}}{2}\right)+\frac{1}{2} \otimes\left(\frac{v_{1}}{2}, 100\right) & v_{1}=v_{2}\end{cases}
$$

where $\frac{1}{2} \otimes\left(100, \frac{v_{1}}{2}\right)+\frac{1}{2} \otimes\left(\frac{v_{1}}{2}, 100\right)$ denotes a $50-50$ mixture between the bid profile $\left(100, \frac{v_{1}}{2}\right)$ and the bid profile $\left(\frac{v_{1}}{2}, 100\right)$.

We now show that $\mu$ constitutes an equilibrium of the second-price auction with communication. Notice first that it is never profitable for a bidder to deviate at the bidding stage when told to bid an amount lower than 100. In this case, the bidder knows the other bidder is bidding 100, and she can only win the object if she bids 100 too, in which case her profit would be at most 0 . Now, suppose bidder $i$ deviates by reporting $\hat{v}_{i}$ and bidding $\hat{b}_{i} \leq 100$ when told to bid 100. If $\hat{b}_{i}<\frac{\hat{v}_{i}}{2}$, she never wins the object and her expected payoff is 0 . If $\hat{b}_{i}>\frac{\hat{v}_{i}}{2}$, her expected payoff is:

$$
\left[\operatorname{Pr}\left(v_{j}<\hat{v}_{i}\right)+\frac{1}{2} \operatorname{Pr}\left(v_{j}=\hat{v}_{i}\right)\right]\left(v_{i}-\frac{\hat{v}_{i}}{2}\right)=\frac{2 v_{i} \hat{v}_{i}-\hat{v}_{i}^{2}}{200}
$$

which is maximized at $\hat{v}_{i}=v_{i}$, in which case bidding $\hat{b}_{i}>\frac{\hat{v}_{i}}{2}$ or 100 generates the same expected payoff. If $\hat{b}_{i}=\frac{\hat{v}_{i}}{2}$, then the bidder receives half of the expected payoff she would receive by bidding 100 , which is not profitable.

The equilibrium $\mu$ implements the same outcome that would have been achieved in the first-price auction without communication. The highest valuation bidder receives the good 
and pays a price that is precisely half of her valuation (when valuations coincide, each bidder gets the good with a 50-50 chance). This completes the proof.

Intuitively, an outcome of the first-price auction can be emulated by the second-price auction as follows. Whenever the bidders are to submit different bids, say $b_{1}>b_{2}$ in the first-price auction, bidder 1 submits 100, thereby assuring she will receive the object, and bidder 2 submits $b_{1}$, thereby assuring the price is $b_{1}$. When bids coincide in the first-price auction, $b_{1}=b_{2}=b$, bidders can toss a fair coin in the second-price auction to determine who will bid 100 and who will bid $b$, which guarantees an equal chance of winning at the price $b$. In the proof we also show that this procedure assures truthful revelation. ${ }^{13}$

The set of equilibrium outcomes in the second-price auction with communication is large. Indeed, bidders can always publicly randomize during the communication phase over which equilibrium they intend to play, assuring that the set of equilibrium outcomes is a convex set. In particular, it contains the convex hull of the outcomes just discussed, those generated by the equilibrium of the first-price auction, as well as the symmetric and asymmetric equilibrium outcomes of the second-price auction (in fact, it strictly contains the convex hull of the set of equilibrium outcomes of second-price auctions without communication). The main message of the proposition is that communication has more of an impact on second-price auctions than it does on first-price auctions.

There are two notes on this theoretical result. First, the cheap-talk extension of the auctions we consider implicitly assumes the availability of an impartial mediator (for the use of the Revelation Principle). The general characterization of games in which unmediated communication generates the same outcomes as mediated communication is a difficult problem (see Gerardi, 2004 and references therein and note that Gerardi, 2004 suggests that with five or more bidders, a mediator is unnecessary). Nonetheless, even absent a mediator, the set of equilibrium outcomes strictly expands when communication is introduced to second-price auctions (for instance, bidders can randomize between equilibria of the auction without communication). Second, we do not preclude weakly dominated strategies. This certainly simplifies the analysis, but the ultimate validity of this allowance is in the data. As will be seen from our experimental results (as well as extant ones for auctions without communication), subjects do not seem to focus on weakly undominated actions.

As described, in some of our treatments we allowed agents to communicate freely prior to bidding and exchange (simultaneously) non-negative transfers after bidding and learning the identity of the object's winner. Formally, the game played is a first- or second-price

\footnotetext{
${ }^{13}$ In fact, the idea of the proof can be extended to auctions with more than two bidders. Furthermore, the inclusion can be extended to general $k$-price auctions. Using the notation in the text, a similar construction can then be used to show that $\Gamma_{k} \subseteq \Gamma_{k+1}$ for all $k \geq 1$.
} 
auction followed by a transfer stage in which agents can simultaneously pick a non-negative number to transfer to the other bidder. Their ultimate payoff is then their payoff in the auction plus the net transfers they have received (the transfers the other bidder passed minus the transfers they had passed to the other bidder). Notice that passing no transfer always strictly dominates passing a positive transfer, regardless of what had transpired in the preceding auction. Therefore, whenever subjects do not use strictly dominated actions at the transfer stage, equilibrium behavior in the treatment with transfers should be consistent with some equilibrium in the cheap-talk extension of our baseline auction before that. ${ }^{14}$ Let $\tilde{\Gamma}_{k}$ denote the set of equilibrium auction outcomes when dominated actions in the transfer stage are not used corresponding to the $k$-price auction with communication and transfers, $k=1,2$. That is, these are mappings from value profiles to distributions over winning probabilities and prices when both communication and transfers are available. We then have the following:

Proposition 2: In any equilibrium of the first- or second-price auctions with communication and transfers in which strictly dominated actions are not used in the transfer stage, no positive transfers are passed. Therefore, the sets of equilibrium outcomes coincide with those of the first- or second-price auctions with communication. That is, $\tilde{\Gamma}_{1}=\Gamma_{1} \subsetneq \tilde{\Gamma}_{2}=\Gamma_{2}$.

To summarize, there are four insights that are relevant to our design. First, without communication, both auction formats entail unique equilibrium predictions when bidders use weakly undominated strategies, and these equilibria are symmetric; The second-price auction entails multiple asymmetric equilibria if the domination restriction is dropped. Second, with communication, second-price auctions generate substantially more equilibrium outcomes than first-price auctions (Proposition 1). In particular, full collusion, associated with 0 revenue, is possible under the second-price auction but not under the first-price auction (Observation 2). Third, effective collusion goes hand-in-hand with a reduction of efficiency (Observation 1). Last, transfers have no impact on outcomes in either auction format and outcomes are predicted to be identical to those in auctions with communication, but without transfers. Furthermore, no positive transfers are passed in any subgame-perfect equilibrium (Proposition 2). ${ }^{15}$

\footnotetext{
${ }^{14}$ We slightly abuse terminology and refer to "actions that are not dominated at the transfer stage." On path, no positive equilibrium transfers occur directly from the definition of best responses. Our restriction therefore has bite only off path.

${ }^{15}$ For simplicity, we assumed a continuous set of values and possible bids. We note, however, that our results do not critically depend on this assumption. Indeed, without communication, Chwe (1989) shows that symmetric equilibria in discrete settings converge to the equilibrium in the continuous setting and our results for the second-price auction remain as they are. Furthermore, the comparison of the two auction formats when communication is available remains intact.
} 


\section{Approach to Data Analysis}

In this section we discuss our approach to data analysis and statistical tests that we use to detect treatment effects and determinants of observed outcomes. The Online Appendix contains many robustness checks relevant to data restrictions discussed below.

First, we focus on rounds 6 through 10 in order to avoid noise due to learning. There are no significant round effects starting from round 6 (see Table 14 as well as Section 5 in the Online Appendix). ${ }^{16}$

Second, the complete strangers sessions, in which subjects never interacted with the same participant more than once, generated data that is statistically indistinguishable from that generated by sessions in which bidders were randomly matched with one another in each round (see Section 2 in the Online Appendix for an analysis of disaggregated data). We therefore report results aggregated across all sessions.

Third, in much of our discussion, we allow for perturbations of 2 experimental points in our classifications. Specifically, we call an outcome efficient if the winning bidder's value is at least as high as the losing bidder's value minus 2 experimental points. We use the term minimal price to refer to a price below 2 experimental points. We call a transfer substantial if the amount transferred is at least 2 experimental points. Finally, we use the same perturbation allowance to define collusive outcomes as well as to classify subjects' announcements regarding their values and bids in the communication stage as truthful, overstated, and understated. In Section 3 of the Online Appendix we show that results remain virtually identical when we consider a smaller perturbation of one experimental point.

Finally, while risk can, in principle, play an important role in bidding behavior, it has no significant effect on neither behavior nor outcomes in our data. We therefore report results without explicitly controlling for elicited risk and refer the reader to Section 4 in the Online Appendix, in which we present the analysis of the impact of risk attitudes that were elicited in several of our sessions.

To compare average outcomes between two groups (be that two treatments, two auctions, or two parts of the experiment), we use regression analysis. More precisely, we run a randomeffects GLS regression in which we regress the variable of interest, e.g. an indicator of whether an outcome is efficient or collusive, on a constant and an indicator for one of the two considered groups. We cluster standard errors by session. We report that there is a significant difference between outcomes in these two groups if the estimated coefficient on

\footnotetext{
${ }^{16}$ We also find no differences in outcomes and behavior observed in sessions that lasted longer than 10 rounds, as is evident from the analysis presented in Section 2 of the Online Appendix.
} 
the group indicator dummy variable is significantly different from zero at the $5 \%$ level.

To compare median outcomes between two groups we use the Wilcoxon rank-sum test and report results at the $5 \%$ significance level. Finally, to compare two distributions (for example, those of prices or those of reported values), we use the Kolmogorov-Smirnov test of equality of distributions and report results at the $5 \%$ significance level.

\section{No Communication}

Recall that absent communication, in our setting, symmetric equilibrium behavior of riskneutral bidders corresponds to each bidder bidding half their object value in the first-price auction and bidding precisely their object value in the second-price auction. ${ }^{17}$ Figure 1 illustrates bidding behavior in our sessions without communication (where, in each panel, the solid line corresponds to the equilibrium bidding strategy). The Figure illustrates that, without communication, our results are in line with the classic observations regarding bidding behavior in experimental auctions (see Cox, Roberson, and Smith, 1982, Dyer, Kagel, and Levin, 1989, Kagel and Levin, 1993, and Harstad, 1991). In first-price auctions, bidders over-bid relative to their equilibrium behavior, but by and large submit bids that are lower than their private valuations. Similarly, in the second-price auction, bidders over-bid as well, submitting bids that are higher than their values a significant fraction of the time. ${ }^{18}$ These trends are reflected in the correlation between bids and values. Clustering observations by session, we find that this correlation is 0.80 in the first-price auction and 1.13 in the second-price auction with robust standard errors of 0.07 and 0.05 , respectively.

In terms of efficiency, we calculate the frequency with which the highest-value bidder wins the object. In our first-price auctions, the mean efficiency is $87 \%$ while in our second-price auctions, the mean efficiency is $77 \%$. These figures are not significantly different from each other at the $5 \%$ level. They mirror the efficiency levels documented in the extant auction literature (see Cox, Roberson, and Smith, 1982 and Kagel and Levin, 1993). We also note that, in our data, inefficiencies are more likely when bidders have values that are close to one another. ${ }^{19}$

\footnotetext{
${ }^{17}$ For second-price auctions, this prediction of behavior does not rely on risk neutrality, but rather on either symmetry or a restriction to weakly undominated strategies.

${ }^{18}$ The use of dominated strategies is not unique to the auction setting. It is observed in many other types of strategic interactions, such as dictator games, trust games, centipede games, etc. (see Kagel and Roth, 1997).

${ }^{19}$ The absolute differences between bidders' values for inefficient outcomes are significantly smaller than those for efficient outcomes at the in both first-price and second-price auctions. In particular, in first-price auctions, the average difference between bidders' values is 30.1 for efficient outcomes and 13.4 for inefficient ones, while in second-price auctions the corresponding values are 37.7 and 22.3 , respectively.
} 
Figure 1: Bidding Patterns in Auctions without Communication
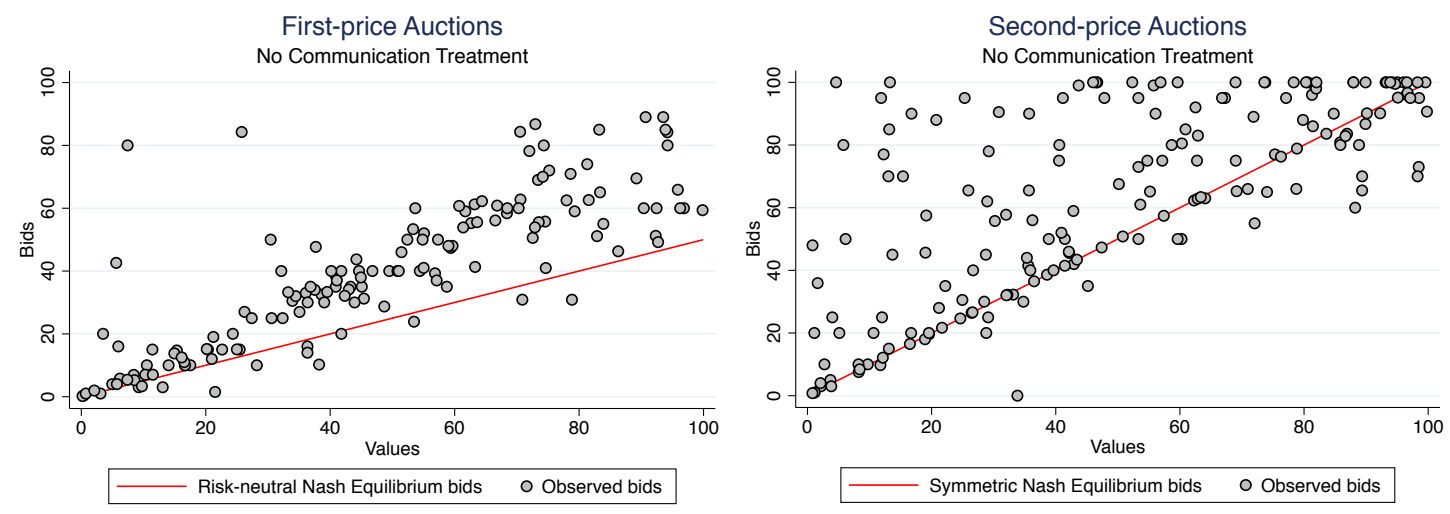

\section{The Emergence of Collusion}

When considering efficiency levels, communication with or without transfers does not generate significantly different outcomes than those generated in sessions without communication. Indeed, in our first-price auctions, generated efficiencies are $82 \%$ and $86 \%$ for the Pure Communication and Communication with Transfers treatments, respectively. Similarly, in our second-price auctions, generated efficiencies are $72 \%$ and $77 \%$ for the Pure Communication and Communication with Transfers treatments, respectively. No pair of values is significantly different at the standard $5 \%$ level.

Figure 2: Cumulative Distribution of Prices across Treatments

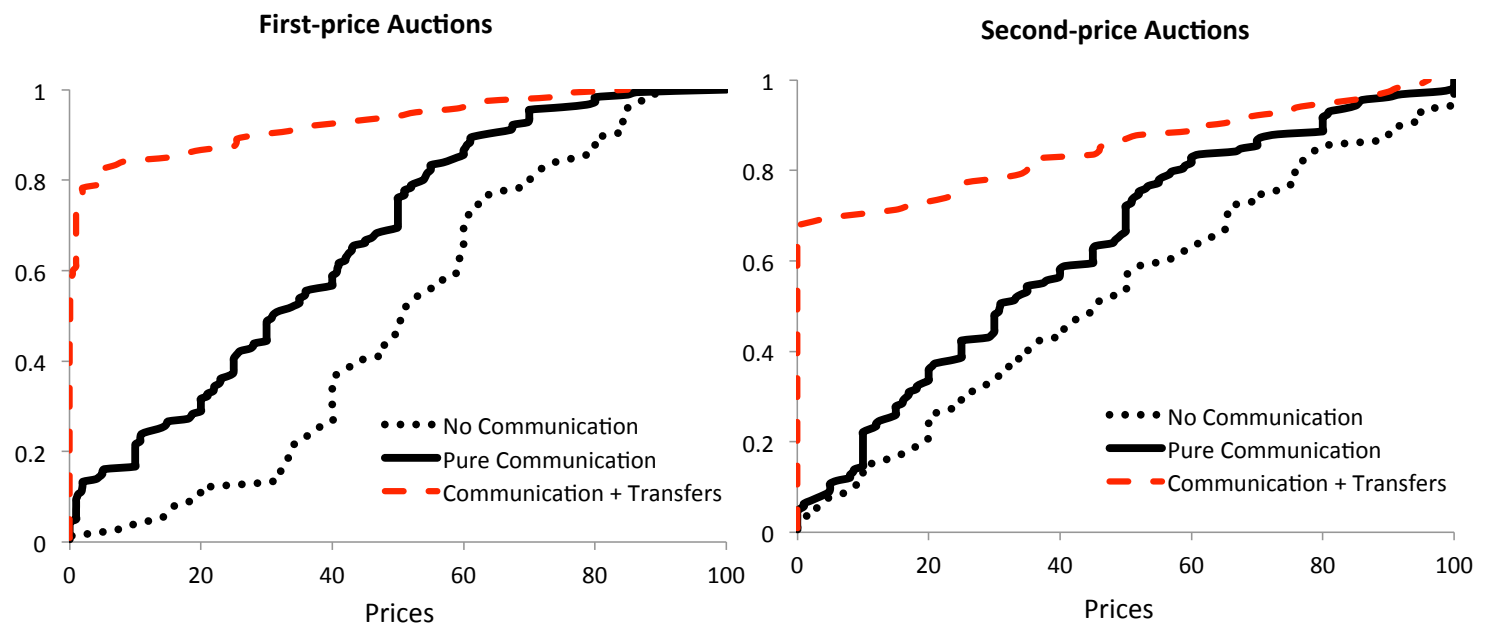

The extent to which our experimental subjects managed to establish successful collusion in our treatments can be seen through the distribution of prices. Figure 2 depicts the cumula- 
tive distribution of prices across our treatments. In both auction formats, price distributions are ranked; the distribution corresponding to our No Communication treatment first-order stochastically dominates that corresponding to our Pure Communication treatment, which first-order stochastically dominates that corresponding to our Communication with Transfers treatments. Kolmogorov-Smirnov test of equality of distributions confirms that price distributions between any two treatments are significantly different at the $5 \%$ level in both auction formats.

These differences in price distributions translate directly to the auctioneer's revenues, as revenues effectively correspond to average prices in our treatments. In our first-price auctions, revenues are 51.2, 33.8, and 7.5 for the No Communication, Pure Communication, and Communication with Transfers treatments, respectively. In our second-price auctions, revenues are 47.8, 38.3, and 15.5 for the No Communication, Pure Communication, and Communication with Transfers treatments, respectively. ${ }^{20}$ Across our treatments, there is a revenue equivalence - the revenues under both auction formats are statistically indistinguishable in all treatments. Importantly, the availability of communication and transfers significantly reduces revenues under both auction formats, generating less than one third of the revenues generated when all communication is banned under either auction format. ${ }^{21}$

As mentioned above, collusion requires one of the participants to submit a low bid so that his or her counterpart will receive the object at a lowered price. Since without communication our results as well as prior literature suggest over-bidding relative to equilibrium behavior, a first glean at collusion can be established by considering the fraction of observations in which the price of the object was below the equilibrium price without communication (namely, half the object's value of the winner in the first-price auction, and the object's value for the losing bidder in the second-price auction). The first column of Table 2 illustrates the frequencies with which such "collusive" outcomes occurred. As can be seen, collusion rates when both communication and transfers were available are very high, $88 \%$ in our first-price auctions and $71 \%$ in our second-price auctions. A harsher definition of collusion corresponds to outcomes that generate minimal prices. The corresponding frequencies appear in the second column of Table 2. Collusion rates in this case are still high and statistically indistinguishable

\footnotetext{
${ }^{20}$ Clustering by session, robust standard errors for the first-price auction revenues are 6.5, 1.9, and 3.0 for the No Communication, Pure Communication, and Communication with Transfers treatments, respectively. For our second-price auction revenues, they are 4.3, 2.7, and 4.6 for the No Communication, Pure Communication, and Communication with Transfers treatments, respectively.

${ }^{21}$ Revenue equivalence across two auction formats and significant decrease in prices in the Pure Communication and Communication with Transfers treatments are confirmed by both regression analysis which compares average prices and Wilcoxon ranksum test which compares median prices. The only exception is the Communication with Transfers treatment, in which median prices in the first- and second-price auctions are different at the $5 \%$ level. In addition, Kolmogorov-Smirnov test rejects equality of price distributions between any two pairs of treatment within the same auction format at the standard $5 \%$ significance level.
} 
Table 2: Frequency of Collusive outcomes across Treatments

\begin{tabular}{lccc}
\hline \hline & $\begin{array}{c}\text { Price is below } \\
\text { Equilibrium Price }\end{array}$ & Price is Minimal & $\begin{array}{c}\text { Price is below Estimated } \\
\text { Price in No Communication }\end{array}$ \\
\hline $\begin{array}{l}\text { First-price Auctions } \\
\text { No Communication }\end{array}$ & & & \\
Pure Communication & $0.05(0.05)$ & $0.00(0.00)$ & $0.58(0.05)$ \\
Communication with Transfers & $0.36(0.07)$ & $0.13(0.04)$ & $0.92(0.03)$ \\
\hline & & $0.78(0.08)$ & \\
Second-price Auctions & & & \\
No Communication & $0.11(0.05)$ & $0.01(0.01)$ & $0.78(0.05)$ \\
Pure Communication & $0.27(0.04)$ & $0.06(0.03)$ & $0.90(0.05)$ \\
Communication with Transfers & $0.71(0.06)$ & $0.68(0.07)$ & \\
\hline \hline
\end{tabular}

Remarks: Price is below Equilibrium Price: observed price is below equilibrium price in the No Communication treatment minus 2 experimental points. Minimal price: a price below 2 experimental points. Price is below Estimated Price in No Communication: observed price is below the estimated price in the No Communication treatment, where the estimated price is based on linear estimations of bidding strategies. Robust standard errors are clustered at the session level and reported in the parenthesis. All calculations of equilibrium prices and estimated prices are done based on the true values of bidders. We exclude auctions in which equilibrium price or estimated price is minimal.

between auction formats, which suggests that most collusive outcomes culminate in a minimal price in the presence of communication and transfers. As we saw, subjects over-bid in our No Communication treatment. Therefore, a weaker definition of collusive outcomes would be one pertaining to outcomes that are below those predicted by the bidding strategies observed when no communication was available. The last column of Table 2 presents the resulting estimates. Numbers are based on estimated linear bidding strategies from our No Communication treatment. For each auction and treatment, we compare the observed price to the price that would have been generated (with the realized values) had subjects used the estimated bidding functions. Under this definition, collusion rates are far higher. Even with communication alone, collusion rates are $58 \%$ in our first-price auctions and $78 \%$ in our second-price auctions. With communication and transfers, collusion rates are at least $90 \%$ in both auction formats. ${ }^{22}$ Taken together, these observations echo those emerging from the price patterns observed in Figure 2: communication alone allows for a significant but limited

\footnotetext{
${ }^{22}$ Statistical analysis reveals that collusion is significantly more frequent in both auction formats when pure communication is available compared to when it is not. Moreover, the availability of transfers in addition to communication increases the instances of collusion even further. All these patterns are significant at the 5\% level under either definition of collusion except for two cases pertaining to our second-price auctions: (1) we detect no statistical difference in collusion rates when pure communication is available compared to when it is not according to our second definition of collusion (second row in Table 2), and (2) we detect no statistical difference in collusion rates when transfers are available compared to auctions in which communication alone was available according to the our last definition of collusion (third column in Table 2).
} 
scope of collusion, while communication and transfers together allow subjects to frequently reduce prices in a substantial way.

In terms of outcomes, the results are not fully in line with the theoretical predictions. The emergence of collusion is not associated with a significant reduction of efficiency (related to our Observation 1). Furthermore, while the theory allows for fully collusive outcomes in second-price auctions, which we observe, it suggests the limited scope of collusion in firstprice auctions (our Observation 2). In our data, both auction formats yield fairly similar outcomes. In the next sections we will see that subjects do respond to some extent to the incentives imposed by first-price auctions to deviate from a collusive outcome. Nonetheless, these responses are not strong enough to maintain the robustness of first-price auctions to communication assured by theory.

\section{Determinants of Prices}

In order to get a sense of the behavior underlying the changes in outcomes generated by the introduction of communication and transfers, we start by analyzing the strategies suggested during the communication stage and followed in the bidding stage of our auctions.

To assure a low price in an auction, at least one of the bidders should submit a low bid (in a first-price auction, both bidders need to submit a low bid for the price to be low). There are indeed two collusive strategies that appear in the analysis of the communication stage. $^{23}$ One strategy profile, which we term the reveal-collude strategy, consists of the bidders revealing their values and submitting bids that assure the object is given to the high-value bidder at a low price, defined as lower than 2. In first-price auctions, this implies both bidders submitting a low bid; In second-price auctions, this implies the losing bidder submitting a low bid and the winning bidder submitting any bid that is higher. The other strategy profile, which we term the flip-a-coin strategy, consists of both bidders submitting the same bid, yielding an equal probability of winning the object for each of the bidders.

Table 3 describes the rates at which each of the strategies was discussed as well as the rates at which each strategy was used. ${ }^{24}$ As can be seen, strategies are discussed at much higher rates when transfers are available. Furthermore, the predominance of strategy discussions when transfers are available focuses on the reveal-collude strategy, while a much

\footnotetext{
${ }^{23}$ Protocols were analyzed by two research assistants, who were not privy to the research questions posed in this paper.

${ }^{24}$ As mentioned, these were the only discussed strategies detected in the communication protocols. Many conversations, particularly in the pure communication treatment, did not involve a discussion of strategy, which we will return to in the next section. We also note that these strategies require a certain level of coordination and were never used unless discussed in the conversation that preceded bidding.
} 
Table 3: Strategies Discussed and Used across Treatments

\begin{tabular}{lcccc}
\hline \hline & \multicolumn{2}{c}{ Pure Communication } & \multicolumn{2}{c}{ Communication with Trasnfers } \\
& First-price & Second-price & First-price & Second-price \\
\hline Discuss Reveal-collude & $6.7 \%$ & $1.9 \%$ & $82.5 \%$ & $70.4 \%$ \\
& & & & \\
Discuss and Use Reveal-collude & $5.0 \%$ & $1.3 \%$ & $66.7 \%$ & $53.0 \%$ \\
Achieved Efficient Outcome & $4.4 \%$ & $1.3 \%$ & $63.3 \%$ & $46.1 \%$ \\
Achieved Inefficient Outcome & $0.6 \%$ & $0.0 \%$ & $3.4 \%$ & $7.8 \%$ \\
Discuss Flip-a-coin & $23.9 \%$ & $4.4 \%$ & $3.3 \%$ & $0.0 \%$ \\
Discuss and Use Flip-a-coin & $3.3 \%$ & $0.6 \%$ & $0.8 \%$ & $0.0 \%$ \\
& & & & \\
\hline \hline
\end{tabular}

Remarks: Discuss Reveal-collude: bidders announce their values and discuss colluding in a way leads the bidder with a higher announced value to win the object. Discuss and Use Reveal-collude: bidders discuss the reveal-collude strategy and the bidder with the higher announced value submits the higher bid, which is lower than 2. Discuss Flip-a-coin: bidders agree to submit the same bid. Discuss and Use Flip-a-coin: bidders discuss the Flip-a-coin strategy and ensuing bids coincide.

higher fraction of strategy discussions focus on the flip-a-coin strategy when no transfers are available.

Both of these strategies are sensitive to deviations by the bidder who is to bid the lowest bid. In the flip-a-coin strategy, both bidders serve in that role and can each increase their bids by a small amount in order to out-bid their opponent. This is reflected in the low rates of incidents of bidders discussing and using this strategy across our treatments. The reveal-collude strategy is assessed as a setting in which at least one of the bidders (both bidders in first-price auctions) submit a bid that is very low. Bidders then might have an incentive to mis-report their values in order to change who is to be winner of the auction. Furthermore, in first-price auctions, a bidder may attempt to out-bid her opponent, while still submitting a low bid to assure the ultimate price is low. Strategic responses as such may both lead to an observed price that is below our threshold of 2. In order to assess when such strategic behavior occurred, we look at the frequency of efficient outcomes when seemingly collusive outcomes were observed. An efficient outcome suggests that the highervalue bidder received the object for a low price; An inefficient outcome suggests that the lower-value bidder received the object at a low price, either due to mis-reporting of her value or due to out-bidding her opponent. As is evident, we see a fairly low fraction of inefficient outcomes when the reveal-collude strategy was carried out, which are mostly our auctions with both communication and transfers. This is suggestive of some but limited scope of strategic manipulation that subjects exercised in this environment. In the next sections we 
inspect bidders' behavior in more detail, both during communication and in the auctions themselves.

Table 4 presents the results of a Tobit regression analysis of how different features of the communication protocols impact prices when bidders interact prior to bidding (errors clustered by session). At the aggregate level, in either auction format, when agents talk during the communication phase prices drop significantly. The topics discussed during communication matter. When transfers are not available, discussion of values does not seem to have a significant effect on prices, discussion of bids does and, in fact, talk of either the flip-a-coin or the reveal-collude strategies has a significant and substantial impact on prices. When transfers are available, discussion of values, bids, or transfers impacts prices significantly and substantially. We note, however, that discussion of bids is highly correlated with discussion of transfers (0.71 in first-price auctions and 0.78 in second-price auctions). Given that their effects on prices are rather similar, it is difficult to isolate which of the two plays a more important role in the determination of the ultimate auction price. We return to a more elaborate analysis of how transfers seem to be set by our subjects in Section 9 .

The fact that discussion of bids, rather than values, seems to be an important force in shaping prices when only communication is available may lead one to wonder about the identity of the winning bidders, whether they are the ones with the highest value or not when prices are low. In other words, are efficiency rates lowered when bidders succeed in colluding? In our Pure Communication treatments, that is indeed the case when considering first-price auctions. Irrespective of the notion of collusion used, we find that efficiency was lower in auctions in which collusive outcomes were achieved compared with auctions in which outcomes were not collusive. ${ }^{25}$ Whether or not a collusive outcome was achieved did not, however, affect efficiency levels in our second-price auctions. One potential explanation for this observation is the following. In first-price auctions, both bidders need to agree to submit a low bid in order for the price to be low. Such a profile is fragile to small deviations. Indeed, suppose two bidders agree that the low-value bidder will submit a bid of 1 and the highvalue bidder will submit a bid of 2 . The low-value bidder need only submit a bid higher than 2 to gain the object (assuming the other remains faithful to the agreement). The price would then remain low, but the resulting allocation will not be efficient. In fact, this type of deviation is at the root of the theoretical result predicting the robustness of first-price auctions to communication. In contrast, in second-price auctions, achieving a low price can

\footnotetext{
${ }^{25}$ In particular, in auctions in which collusive outcome was achieved, efficiency levels were $0.63,0.43$ and 0.75 for the first, the second and the third definition of collusion (which correspond to the first, the second and the third column in Table 2), respectively, while for auctions in which outcomes were not collusive, efficiency levels were $0.92,0.87$ and 0.91 , respectively.
} 
Table 4: Tobit Estimates Explaining Ultimate Prices

\begin{tabular}{|c|c|c|c|c|c|c|c|c|c|}
\hline & \multicolumn{9}{|c|}{ First-price Auctions } \\
\hline & \multicolumn{4}{|c|}{ Pure Communication } & \multicolumn{5}{|c|}{ Communication with Transfers } \\
\hline & Regression 1 & Regression 2 & Regression 3 & Regression 4 & Regression 1 & Regression 2 & Regression 3 & Regression 4 & Regression 5 \\
\hline Equilibrium Prediction & $1.0^{* *}(0.1)$ & $0.8^{* *}(0.04)$ & $0.8^{* *}(0.1)$ & $0.8^{* *}(0.03)$ & $0.1(0.1)$ & $0.04(0.04)$ & $0.1(0.1)$ & $0.1(0.1)$ & $0.1(0.1)$ \\
\hline Indicator if & & & & & & & & & \\
\hline $\begin{array}{l}\text { Bidders Talk } \\
\text { Indicator if Bidders }\end{array}$ & $-19.9^{* *}(2.5)$ & & & & $-41.7^{* *}(12.4)$ & & & & \\
\hline $\begin{array}{l}\text { Indicator if Bidders } \\
\text { Discuss Values }\end{array}$ & & $2.54(3.24)$ & & & & $204 * *$ & & & \\
\hline Indicator if Bidders & & $2.04(0.24)$ & & & & $-30.4 \quad(11.0)$ & & & \\
\hline Discuss Bids & & & $-25.6^{* *}(2.8)$ & & & & $-27.3^{* *}(5.7)$ & & \\
\hline Indicator if Bidders & & & & & & & & & \\
\hline Discuss Flip-a-coin & & & & $-25.4^{* *}(3.5)$ & & & & $-30.9^{* *}(5.4)$ & \\
\hline Indicator if Bidders & & & & & & & & & \\
\hline Discuss Reveal-collude & & & & $-21.8^{* *}(4.8)$ & & & & $-29.0^{* *}(5.9)$ & \\
\hline Indicator if Bidders & & & & & & & & & \\
\hline Discuss Transfers & & & & & & & & & $-26.6^{* *}(4.2)$ \\
\hline Constant & $16.1^{* *}(2.3)$ & $0.3(4.8)$ & $16.1^{* *}(3.6)$ & $12.1^{* *}(1.8)$ & $42.3^{* *}(14.2)$ & $33.1^{* *}(10.7)$ & $26.1^{* *}(3.8)$ & $27.7^{* *}(5.4)$ & $24.9^{* *}(5.0)$ \\
\hline \# of obs & 180 & 130 & 130 & 134 & 120 & 112 & 112 & 112 & 112 \\
\hline \# of sessions & 6 & 6 & 6 & 6 & 4 & 4 & 4 & 4 & 4 \\
\hline \multirow[t]{4}{*}{ Pseudo $\mathrm{R}^{2}$} & 0.0463 & 0.0193 & 0.0727 & 0.0684 & 0.0505 & 0.0173 & 0.0658 & 0.0504 & 0.0453 \\
\hline & \multicolumn{9}{|c|}{ Second-price Auctions } \\
\hline & \multicolumn{4}{|c|}{ Pure Communication } & \multicolumn{5}{|c|}{ Communication with Transfers } \\
\hline & Regression 1 & Regression 2 & Regression 3 & Regression 4 & Regression 1 & Regression 2 & Regression 3 & Regression 4 & Regression 5 \\
\hline Equilibrium Prediction & $0.9^{* *}(0.1)$ & $0.9^{* *}(0.2)$ & $0.8^{* *}(0.1)$ & $0.8^{* *}(0.1)$ & $0.6^{* *}(0.2)$ & $0.7^{* *}(0.3)$ & $0.7(0.5)$ & $0.7^{* *}(0.3)$ & $0.7^{* *}(0.3)$ \\
\hline Indicator if & & & & & & & & & \\
\hline Bidders Talk & $-9.6^{* *}(1.9)$ & & & & $-71.6^{* *}(18.5)$ & & & & \\
\hline Indicator if Bidders & & & & & & & & & \\
\hline Discuss Values & & $9.0(6.3)$ & & & & $-75.9^{* *}(20.3)$ & & & \\
\hline Indicator if Bidders & & & & & & & & & \\
\hline Discuss Bids & & & $-26.5^{* *}(6.1)$ & & & & $-66.6^{* *}(15.6)$ & & \\
\hline Indicator if Bidders & & & & & & & & & \\
\hline Discuss Flip-a-coin & & & & $-26.3^{* *}(5.2)$ & & & & & \\
\hline Indicator if Bidders & & & & & & & & & \\
\hline Discuss Reveal-collude & & & & $-27.2^{* *}(12.2)$ & & & & $-76.2^{* *}(15.7)$ & \\
\hline Indicator if Bidders & & & & & & & & & \\
\hline Discuss Transfers & & & & & & & & & $-63.8^{* *}(11.8)$ \\
\hline Constant & $10.4^{* *}(3.2)$ & $-5.2(5.5)$ & $10.3(6.0)$ & $6.7(5.2)$ & $26.3^{* *}(11.7)$ & $11.4(12.1)$ & $-5.4(23.1)$ & $9.7(15.8)$ & $-7.0(20.5)$ \\
\hline \# of obs & 143 & 44 & 44 & 50 & 113 & 97 & 95 & 98 & 98 \\
\hline \# of sessions & 5 & 5 & 5 & 5 & 4 & 4 & 4 & 4 & 4 \\
\hline Pseudo $\mathrm{R}^{2}$ & 0.0847 & 0.0701 & 0.1248 & 0.1172 & 0.0611 & 0.0563 & 0.0671 & 0.1139 & 0.0787 \\
\hline
\end{tabular}

Remarks: We run Tobit regressions, in which we regress Price on the equilibrium price predicted by risk-neutral Nash equilibrium price and indicators capturing conversations between bidders. Robust standard errors are clustered at the session level. Reveal-collude is the strategy in which bidders announce their values and discuss colluding in a way that a bidder with a higher announced value bids higher than the other one. Flip-a-coin is the strategy in which bidders agree to put the same bid. 
entail the intended winner of the auction submitting a high bid (potentially the maximal possible bid of 100). There are therefore agreements that assure that a deviation of the intended low bidder cannot gain him or her the object at a low price. Our analysis of the communication protocols in the following section echoes this discussion.

\section{Analysis of Communication}

We now turn to the analysis of the communication protocols themselves in the sessions that allowed for some interaction between bidders. We start by describing the frequencies with which bidders conversed about values and bids and the extent to which they misrepresented their reports. We then analyze the dynamics within the protocols that emerged.

The left panel of Figure 3 illustrates the fraction of subjects who did not talk, talked and overstated their values or bids, revealed truthfully their values or bids, or understated their values or bids. In this figure we treat each subject in each round (of a particular auction format) as an independent observation. ${ }^{26}$ As can be seen, in the majority of auctions subjects do not talk about some aspect of the auction when only communication is available. In our first-price auctions $68 \%$ of subjects chose not to talk about their bids and $67 \%$ of subjects chose not to talk about their values. The frequency of communication in our second-price auctions with pure communication is even lower: over $88 \%$ of subjects did not discuss their values and $93 \%$ did not discuss their bids. Conditional on talking, subjects mis-report quite frequently, in fractions comparable to those corresponding to truth-telling. Furthermore, the most prominent pattern of lying is to understate one's true value or intended bid.

\subsection{Topics of Conversation and Misrepresentation}

Figure 4 depicts the distribution of announced values and bids as a function of actual values and bids, respectively (where the size of each point is a proxy for the corresponding number of observations) in our Pure Communication treatments. With respect to values, there is substantial volume of truth-telling, as well as some over-statement of values and more frequent under-statement of values. With respect to bids, much of the truthful revelation is linked with fairly low bids, as is much of the misrepresentation. In fact, as we discussed in Section 7, many of the subjects attempt to collude by suggesting a low bid; for instance, by using the flip-a-coin strategy. Nonetheless, many subjects understand that both subjects

\footnotetext{
${ }^{26}$ Values and bids were communicated as numbers or verbally ("low", "high", etc.). The two research assistants that analyzed the communication protocols were instructed to interpret "low" and its synonyms as corresponding to 0-33, "moderate" and its synonyms to 34-66, and "high" and its synonyms to 67-100. These categories allow us to classify the lying patterns for subjects using verbal messages as well.
} 
Figure 3: Frequency of Communication across Treatments
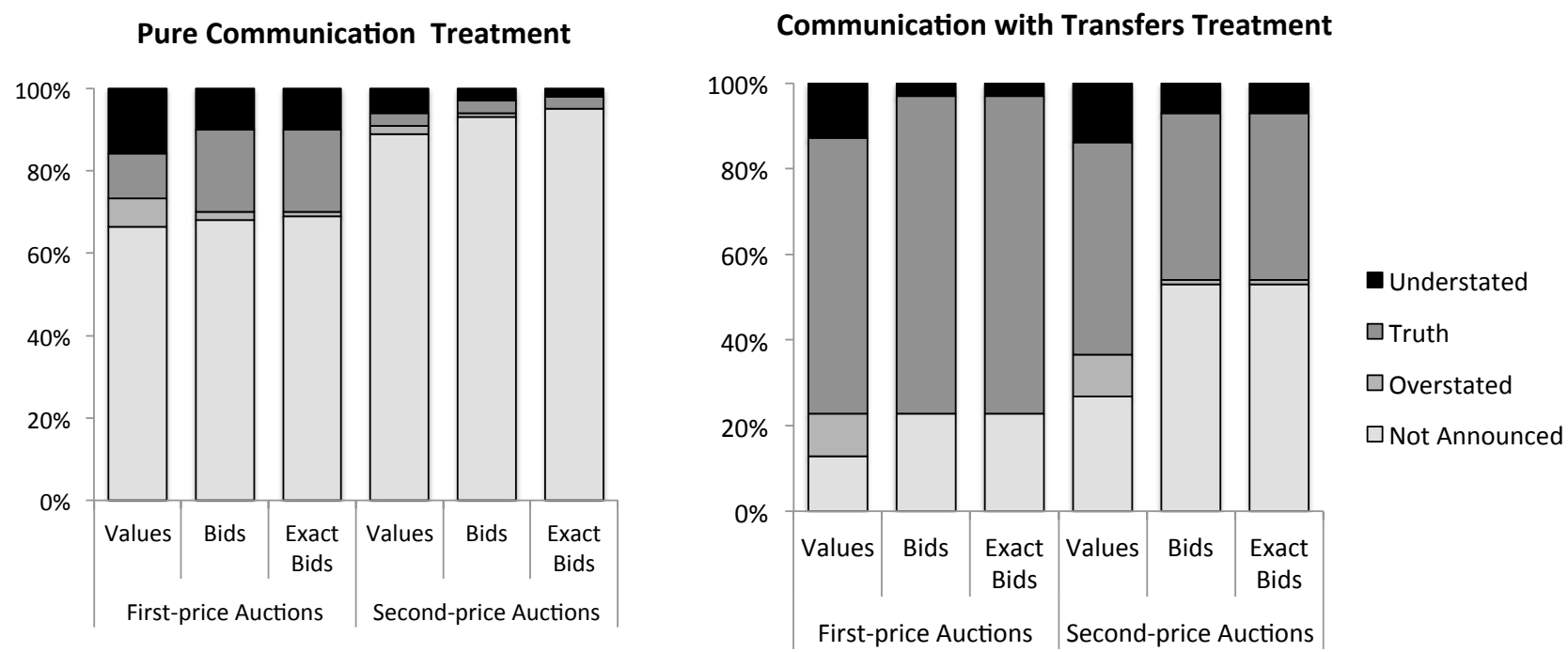

Remarks: Values and Bids refer to both numerical and verbal descriptions of values and bids. Exact Bids refers only to numerically stated bids. For each category, we classify announced values and bids relative to the actual values and bids. The value (bid) is understated if the announced value (bid) is below the actual value (bid) minus 2 experimental points. The value (bid) is overstated if the announced value (bid) is above the actual value (bid) plus 2 experimental points. Finally, if the announced value (bid) is between the actual value (bid) minus 2 and the actual value (bid) plus 2, then it is classified as Truth. Verbal announcements were of the form "low," "moderate," and "high," which we interpret as 0-33, 33-67, and 67-100, respectively. We used the average announcements of these ranges to classify announcements as those that are overstated, those that are understated, and those that are truthful. 
Figure 4: Misrepresentation Patterns in Pure Communication Treatments

First-price Auctions Pure Communication Treatment
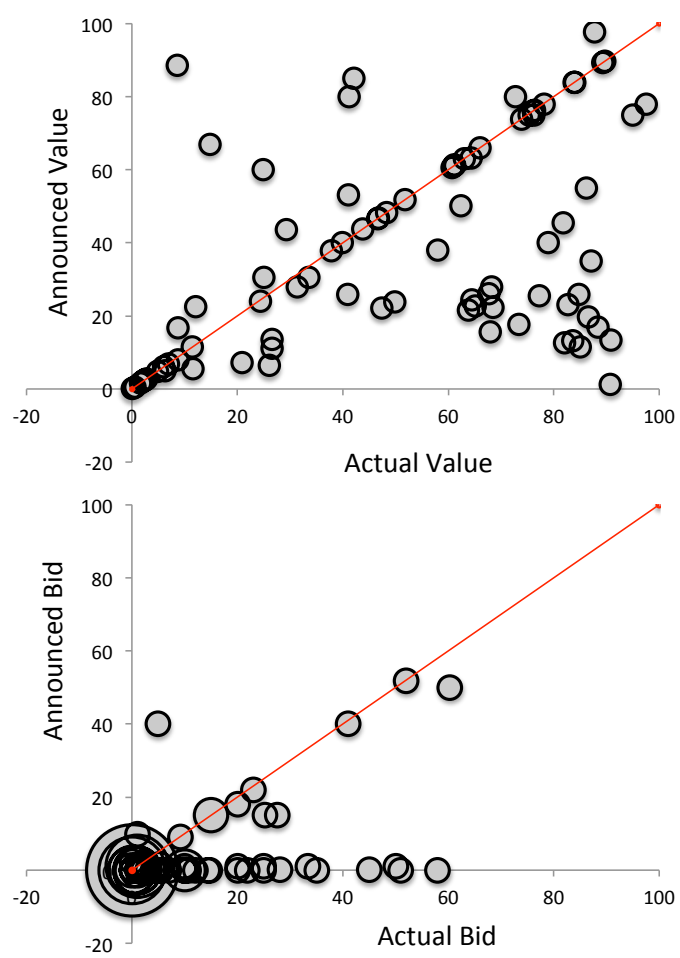

Second-price Auctions Pure Communication Treatment
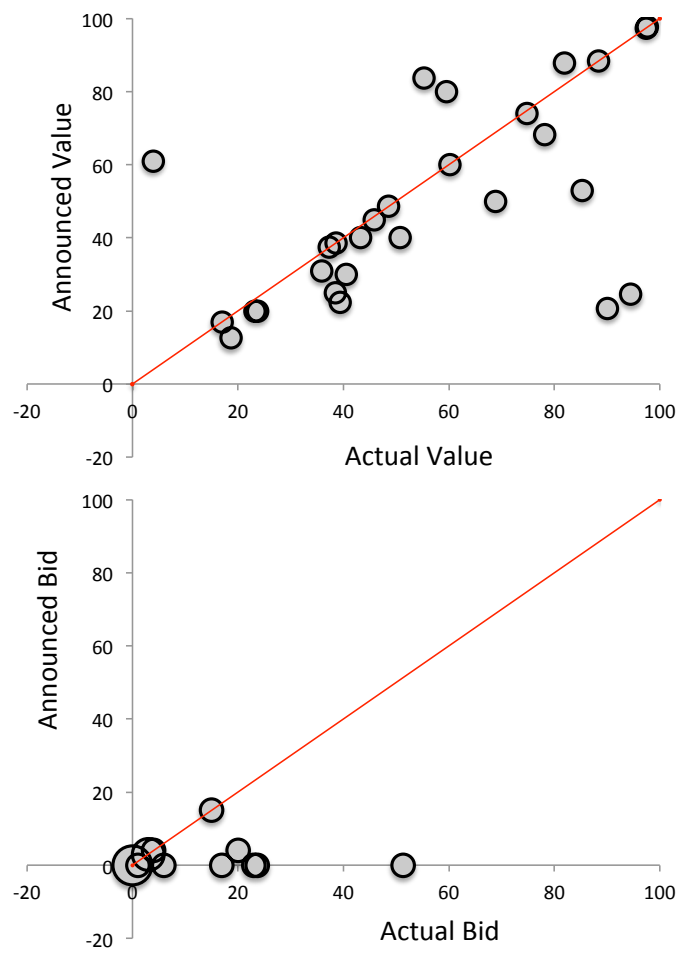
bidding low is not incentive compatible - particularly in the first-price auction, where both individuals need to announce a low bid for collusion to have a chance, a bidder can bid slightly over the announced bid and still get the object for a fairly low price. ${ }^{27}$ This is in line with the theoretical forces that, in principle, should make first-price auctions robust to communication. In our experiments, subjects are fairly successful at generating collusive outcomes even in first-price auctions, but the different strategic incentives may explain the somewhat different patterns we observe in reporting between the two auction formats.

In contrast, the right panel of Figure 3 illustrates that the cheap-talk channel is used very differently in treatments with communication alone and in treatments in which transfers were also available. The first distinction is the frequency with which subjects discuss relevant things prior to the auction: while in our Pure Communication treatments these discussions were rare, they are prevalent in the treatments with transfers under both auction formats. Indeed, subjects talk about their values in $87 \%$ of the cases and about their bids in $77 \%$ of the cases in our first-price auctions. In our second-price auctions they discuss values in $73 \%$ of the cases and bids in $47 \%$ of the cases. Furthermore, while in the Pure Communication treatment, conditional on talking, most of the revealed values and bids were attempts to misrepresent the truth, this is not the case in the Communication with Transfers treatment, in which most of the reports are truthful. Conditional on reporting a value, subjects report truthfully $75 \%$ of the time in our first-price auctions and $68 \%$ of the time in our second-price auctions. Similarly, conditional on reporting one's intended bid, subjects follow through with ther intention $97 \%$ of the time in our first-price auctions and $83 \%$ of the time in our second-price auctions.

\subsection{Dynamics of Communication Protocols}

Protocols were very short - between three and six messages in the Pure Communication treatment and between five and six messages in the Pure Communication with Transfers treatment. ${ }^{28}$ Nonetheless, we can identify several consistent patterns in the protocols we observe.

\footnotetext{
${ }^{27}$ In first-price auctions, the two most common reported bid and actual bid pairs are $(0,0)$ and $(0, \varepsilon)$, where $\varepsilon=0.01$ or $\varepsilon=1$ (indicated by the two big circles on the bottom-left panel of Figure 4$)$. The $(0, \varepsilon)$ case represents bidders who reported that they will bid 0 but then deviated to a bid of $\varepsilon>0$ in an attempt to game the opponent and secure winning the auction. In most of these cases, subjects discussed and agreed to play the flip-a-coin strategy, and usually one of the bidders followed through on his or her promise (which is indicated by the substantial number of observations of $(0,0))$.

${ }^{28}$ In the Pure Communication treatment, the average number of messages sent was 5.6 in our first-price auctions and 3.5 in our second-price auctions (with standard errors of 0.4 and 0.7 , respectively). In our Communication with Transfers treatment, the average number of messages sent was 5.8 in our first-price auctions and 5.2 in our second-price auctions (with standard errors of 0.3 for both).
} 
At the aggregate level, individuals are significantly more likely to announce their values or bids when their counter-part has already done so. ${ }^{29}$ While in our Communication with Transfers treatments most reports are truthful, the order in which announcements are made in our Pure Communication treatment affects the announcements themselves. Bidders who speak first about values announce a lower value than those who speak second about their value, albeit not significantly so. On the contrary, bidders who speak first about their intended bid announce a higher bid than those who speak second, though these differences are not statistically significant either. We note, however, that in all our sessions the order in which values or bids are communicated does not seem to explain outcomes. For example, the first to reveal his or her value had an approximately 50-50 chance of winning the object. Furthermore, the length of conversations, in terms of time or number of messages, does not seem to predict outcomes, including efficiency and collusion rates.

\section{Figure 5: Dynamics of Communication Protocols}

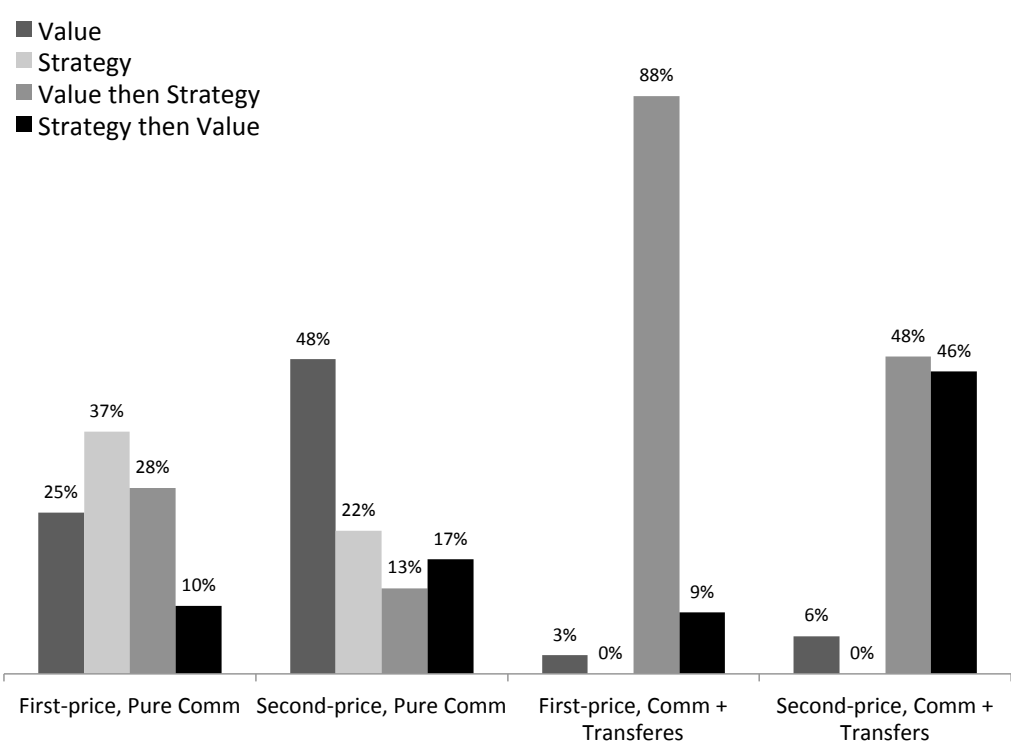

Figure 5 depicts the order that protocols followed in our treatments. There are two important dimensions that are discussed during communication - values and strategies, which encompass either bids or transfers. In our Communication with Transfers treatment, discussions of bids and transfers went hand-in-hand (conditional on discussing bids, in $94 \%$

\footnotetext{
${ }^{29}$ We used random effects Probit regressions to explain either a report of value or a report of bid with one's own value, and whether the other bidder announced a value or bid, while clustering observations by session. When looking at value reports, the strongest explanatory variable is the announcement of value by the other bidder. Similarly, when looking at bid reports, the strongest explanatory variable is the announcement of bid by the other bidder.
} 
or more auctions of either type bidders discussed transfers as well). Therefore, we do not separate instances of bid discussion from those in which transfers were discussed. The figure illustrates when only values or only strategies were discussed, and if both were discussed, what order they followed. As can be seen, in our Pure Communication treatments, no clear pattern emerges, though in second-price auctions the modal protocol entails a discussion of values alone. However, when transfers are introduced, subjects discuss both values and strategies at very high rates and, particularly in our first-price auctions, a discussion of values often precedes that of strategy.

\section{The Impacts of Transfers}

Our treatment with both communication and transfers seems to have been the most effective in allowing subjects to collude in both auction formats. This is despite the fact that subjects could not commit to the transfers passed and were randomly matched with one another to prevent effective commitment through repeated play. In the Online Appendix (Tables 19 and 20) we present additional analysis that indicates this is also true in the very last rounds of our sessions, as well as in the sessions in which a complete-strangers matching protocol was used, in which subjects knew they would never encounter the same partner more than once. We first note that transfers rarely originate from the loser of the object to its winner; This occurred in fewer than $5 \%$ of cases under both auction formats. Winners, on the other hand, transfer more than 2 points to their losing opponents at a frequency of $65 \%$ in our first-price auctions and $48 \%$ in our second-price auctions. The frequency of transfers depends on how the auction culminates: transfers are very frequent if the ultimate price is minimal (the frequency of substantial transfers in our first-price auctions is $79 \%$ while it is $71 \%$ in our second-price auctions). ${ }^{30}$ However, if the price above minimal, then substantial transfers are rare (15\% in our first-price auctions and $0 \%$ in our second-price auctions). ${ }^{31}$

\footnotetext{
${ }^{30}$ Our setting is reminiscent of trust games in that losing bidders need to forgo the chance to win the object and submit a low bid providing their opponents large profits in the hope that money will be passed back to them. As a comparison, Berg, Dickhaut, and McKabe (1995) studied trust games in which the first player had $\$ 10$ to allocate and any amount passed on to the second player was multiplied by 3. Of 28 pairs in which some money was transferred, in 12 cases (or 43\%) money was transferred back, in line with our figures.

${ }^{31}$ We note that these high frequencies of positive transfers from the winning bidders to the losing bidders remain prevalent even in the very last round of the experiment. Indeed, in $71 \%$ of cases, in the last round of our first-price auctions winners transferred substantial amounts to their losing counterpart, while this happened in $85 \%$ of cases in which the price was minimal. Similarly, in the very last round of our secondprice auctions, in $48 \%$ of the auctions winning bidders transferred substantial amounts to the losing bidders, while this fraction becomes $65 \%$ once we condition on the price of the object being minimal. These data reinforce the claim that our observations are not driven by wrong perceptions of repeated play, since subjects knew when the last round of the experiment was taking place.
} 
Table 5: Probit Estimates Explaining when Transfers Occur

\begin{tabular}{lcc}
\hline \hline & First-price Auctions & Second-price Auctions \\
\hline Winning Bidder's Value & $0.01^{* *}(0.005)$ & $0.01^{* *}(0.007)$ \\
Losing Bidder's Value & $-0.002(0.004)$ & $-0.005(0.008)$ \\
Price & $-0.04^{* *}(0.01)$ & $-1.39^{* *}(0.62)$ \\
Indicator if Efficient Outcome & $1.31^{*}(0.78)$ & $0.87^{* *}(0.44)$ \\
$\quad$ Indicator if Winning Bidder Lied & & \\
$\quad$ in the Past about Values or Bids & $-0.12(0.11)$ & $-0.45^{*}(0.25)$ \\
Constant & $-1.16^{*}(0.56)$ & $-0.54(0.35)$ \\
& & \\
\# of obs & 120 & 115 \\
\# of sessions & 4 & 4 \\
Pseudo R-square & 0.3126 & 0.5229 \\
\hline \hline
\end{tabular}

Remarks: Estimates are from the Probit regressions with a dependent variable that takes the value of 1 if the winning bidder transferred a substantial amount (at least 2 experimental points) to the losing bidder and zero otherwise. Robust standard errors are clustered at the session level. ${ }^{* *}\left({ }^{*}\right)$ indicates significance at the $5 \%(10 \%)$ level.

Table 5 reports results from a Probit regression in which whether or not substantial transfers were passed is explained by the winning and losing bidders' values, the ultimate price in the auction (a proxy for whether a collusive outcome emerged), an indicator that takes a value of 1 if the auction culminated in an efficient outcome, and an indicator that takes a value of 1 if the winning bidder had lied in the previous auctions about his or her values or bids. The last variable is designed to capture a "type" of bidder that is more inclined to try acting in a self-interested manner.

As can be seen, the winning bidder's value and the final price have a significant impact on whether transfers occur, with price having a larger effect than the winning bidder's value. ${ }^{32}$ The losing bidder's value has no significant effect on whether or not transfers occur. Efficient outcomes are associated with a significantly higher likelihood of transfers being passed. Note that collusive outcomes require some coordination achieved through communication and efficiency is a proxy for whether values are revealed truthfully. Nonetheless, the winning bidder's truthfulness in prior rounds, which could serve as a proxy for her "type," does not seem to play an important role in the occurrence of transfers. We do see some difference between the two auction formats in terms of the impact of final prices: they exhibit a larger effect in our second-price auctions.

\footnotetext{
${ }^{32}$ Using the winning bidder's surplus instead of these two variables leads to a similar conclusion - a higher surplus is linked with a greater likelihood of transfers occurring.
} 
Conditional on making a substantial transfer, the amount transferred averages at $44 \%$ of the surplus in our first-price auctions and $45 \%$ of the surplus in our second-price auctions. However, the modal fraction of surplus transferred is $50 \%$ - in $55 \%$ of our first-price auctions and $67 \%$ of our second-price auctions half of the surplus was transferred. In line with our observations above, the amount transferred does not depend on the losing bidder's value. In Section 11.1 we discuss how norms prescribing such giving behavior might make the behavior we observe consistent with equilibrium play.

These observations suggest a consistent pattern of behavior as follows. Subjects share some (mostly truthful) information regarding their values during communication. They then submit bids that assure a fairly low price and realize approximately the maximal surplus for the bidders given their values. Last, they share the surplus at roughly equal proportions.

\section{Repeated Interactions with and without Commu- nication}

Since much of the theoretical literature studying collusion in auctions has focused on repeated interactions as a driving channel, we ran several auxiliary sessions to assess the impact of repeated interactions and compare them with our benchmark one-shot setting.

\subsection{Design of Auxiliary Sessions}

In each of our auxiliary sessions, subjects were initially randomly paired. They then proceeded to play a repeated game in which each stage mimicked our one-shot setting. That is, subjects were informed of their private values, submitted simultaneously their bids, and were informed of the outcome of the auction. The repeated game terminated with a $10 \%$ probability in each period. This probability was chosen so that the expected number of rounds played will be 10, the number of rounds in the sessions of our benchmark treatments. In addition, with a 10\% termination probability, there is a fully collusive equilibrium under both the first- and second-price repeated auction in which, on path, prices are 0. For example, in our repeated first-price auction, a profile in which both bidders submit a bid of 0 (thereby leading to a probability of $1 / 2$ that each bidder wins the auction in each period) and switch to the one-shot equilibrium profile upon a deviation constitutes an equilibrium. In our second-price auction, a profile in which bidders alternate between who bids 0 and who wins the object (at a resulting price of 0 ) and switch to the one-shot equilibrium profile upon a deviation constitutes an equilibrium. We note that this latter sort of alternating equilibrium exists also in our first-price auction, with a slight modification. Namely, in each round, 
one subject would submit a bid of 0 , while the other would submit the minimal positive bid (corresponding to 0.01 experimental points).

In order to assure we observe the behavior of subjects for at least 10 rounds, subjects all played the first 10 rounds (regardless of whether the game had terminated or not). If the game terminated within the first 10 rounds, we informed subjects that was the case and this marked the end of the session. Subjects were then paid for all periods in which the game was active. ${ }^{33}$ If the game had not terminated, subjects continued playing as long as the game was active. However, after round 10, we informed subjects in each round whether the game had come to an end or not.

We ran two sessions as above, one corresponding to the first-price auction (with 24 subjects, yielding 12 independent pairs) and one corresponding to the second-price auction (with 30 subjects, yielding 15 independent pairs).

In addition, we ran analogous sessions in which, in every round, subjects could communicate freely as in our benchmark sessions after observing their private values and before submitting their bids. We ran one session as such for the first-price auction (with 26 subjects, yielding 13 independent pairs) and one for the second-price auction (with 30 subjects, yielding 15 independent pairs). ${ }^{34}$

In what follows, unless otherwise mentioned, we focus on rounds 6-10, so that results are comparable with those pertaining to our benchmark setting.

\subsection{Efficiency and Collusion in Repeated Auctions}

Without communication, efficiency levels in our repeated auction setting are similar to those corresponding to our one-shot setting. In our repeated first-price auctions, the mean efficiency is $93 \%$, while in our second-price auctions, it is $88 \%$. However, in contrast with our benchmark setting, the introduction of communication reduces substantially the levels of efficiency. In our repeated first-price auctions with communication, the mean efficiency level is $77 \%$, while it is only $63 \%$ in our repeated second-price auctions with communication. This is consistent with the fraction of collusive outcomes being generated by simple strategies in the repeated game that lead to winning bidders alternating or being randomly determined. We return to the patterns of behavior we observe in the next subsection.

With respect to prices, Figure 6 depicts the cumulative distributions of prices across all our treatments. As can be seen, repeated interaction, with or without communication, does not enlarge the scope of collusion relative to our one-shot treatment with communication

\footnotetext{
${ }^{33}$ For instance, if the game terminated after period 5 , subjects were paid for the first 5 periods only.

${ }^{34}$ All sessions other than the one corresponding to our repeated first-price auction treatment terminated within the first 10 rounds. The repeated-first price auction session terminated only in period 20 .
} 
and transfers. In fact, in our repeated first-price, distribution of prices with or without communication first order stochastically dominate the distribution we observed in our oneshot treatment with communication and transfers; In our repeated second-price auctions, the price distribution when communication is available is first order stochastically dominated by that corresponding to the case in which communication was not available, however it virtually coincides with the distribution of prices in our one-shot second-price auction with communication and transfers. ${ }^{35}$ This leads to an interesting distinction between our one-shot auctions and our repeated ones: unlike our one-shot auctions, repeated first- and second-price auctions generate significantly different outcomes. We now shift to describing the behavior in these auction, which underlies the difference.

Figure 6: Cumulative Distributions of Prices across Treatments

First-price Auctions

Second-price Auctions
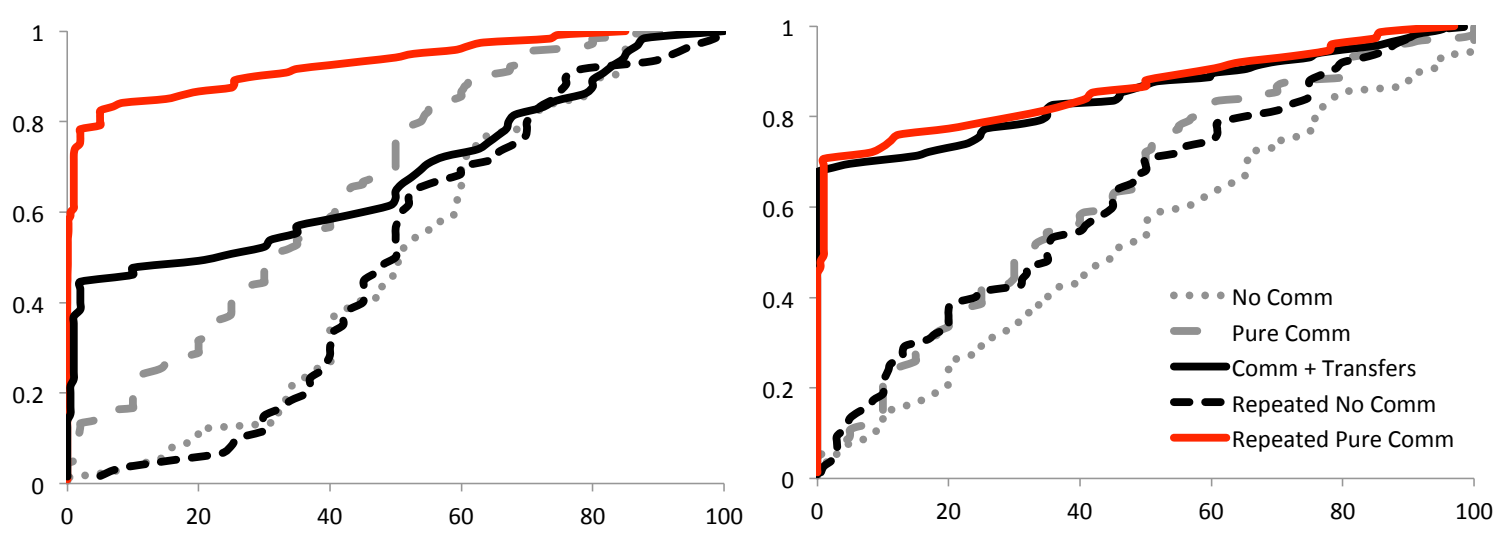

\subsection{Behavior in Repeated Auctions}

Beyond the resulting distribution of prices that we discussed above, one way to glean some intuition as to the patterns of behavior in our repeated auctions can be achieved by looking at the sequences of consecutive wins of any subject. Define a run as a maximal block of rounds in which one bidder won. We define the number of switches in a session to be the number of times the identity of the winning bidder changed in consecutive rounds. The number of switches is the number of runs minus 1. For example, if one bidder wins the

\footnotetext{
${ }^{35} \mathrm{~A}$ series of Wilcoxon ranksum tests confirm that median prices observed in our repeated first-price auctions with or without communication are significantly higher than those observed in our one-shot firstprice auctions with communication and transfers at the $5 \%$ level. At the same time, we find no statistical difference between median prices observed in our repeated second-price auctions with communication and those observed in our one-shot second price auctions with communication and transfers.
} 
auction throughout a session of 10 rounds, there would be only one run and no switches; If bidders alternate who wins across rounds, the maximal number of 10 runs and 9 switches would be observed. The number of switches is then a proxy for the extent to which subjects alternate between who is the winning bidder. If the winning bidder's identity were random (as would be the case if outcomes were fully efficient), the expected number of runs would be 5.5 leading to an expected 4.5 switches. $^{36}$

Figure 7 depicts the distribution of the number of switches of winning bidders in our different repeated auctions. As can be seen, there is a substantially higher number of switches when communication is possible. In fact, absent communication, the distribution of switches looks fairly random, with an average of 4.5 switches in our repeated first-price auctions and 4.7 switches in our repeated second-price auctions, indistinguishable from the expected number of switches were winners determined randomly. With communication, the average number of switches increases to 5.9 in our repeated first-price auctions and 5.7 in our repeated second-price auctions and we see a significant fraction of groups exhibiting 8 or 9 switches. ${ }^{37}$ These observations suggest that at least some of the collusive outcomes we observe when communication is available in our repeated auctions comes hand in hand with subjects attempting to alternate between who wins the auction.

Figure 7: Distribution of Number of Switches of Winning Bidders

First-price Auctions

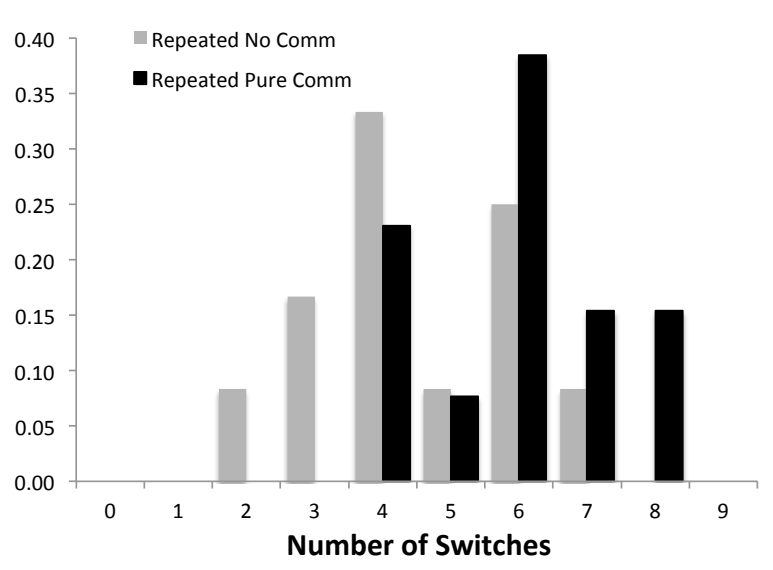

Second-price Auctions

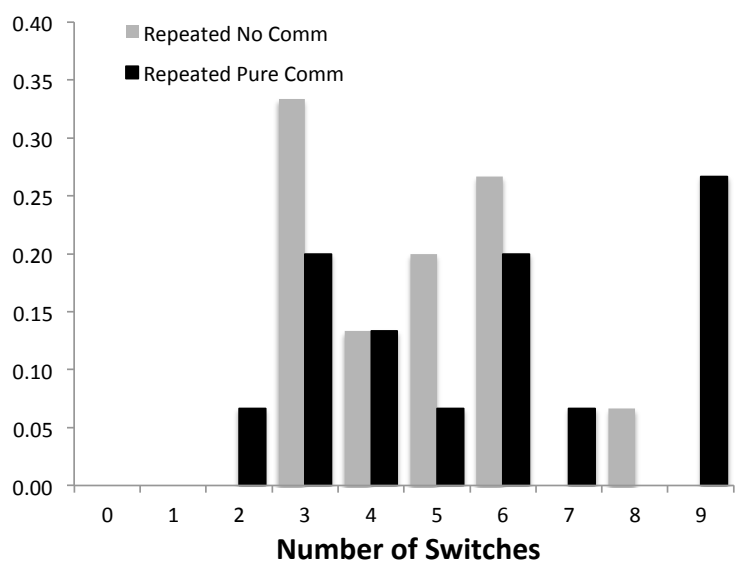

\footnotetext{
${ }^{36}$ Indeed, denote by $Z_{t}$ for $t=2, \ldots, 10$ an indicator that takes the value of 1 if the winner in round $t$ is different than the winner in round $t-1$. Notice that $\mathbb{E}\left(Z_{t}\right)=1 / 2$. The number of runs is then $1+\sum_{t=2}^{n} \mathbb{E}\left(Z_{t}\right)=5.5$ and the number of switches is $\sum_{t=2}^{n} \mathbb{E}\left(Z_{t}\right)=4.5$.

${ }^{37}$ The probability of 9 switches when winners are determined randomly is $\frac{1}{2}^{9}$, lower than $0.2 \%$. The probability of 8 switches when winners are determined randomly is $9 \cdot \frac{1}{2}^{9}$, lower than $2 \%$.
} 
The protocols of communication allow us to inspect the potentially intended strategy profiles of subjects. In our first-price repeated auctions with communication, 8 of the 13 groups (or 62\%) had discussions relevant to the auction carried out. In our second-price repeated auctions, 12 out of 15 (or $80 \%$ ) had such discussions. $75 \%$ of the groups under both auction formats discussed, at some point during the session, an alternating-turns strategy under which bidders alternate between who wins the object (which, as we noted above, can constitute part of an equilibrium). $38 \%$ of the groups in our first-price auctions and $42 \%$ of the groups in our second-price auctions discussed the reveal-collude strategy at some point. ${ }^{38}$

Whether or not groups meaningfully communicated substantially affected the extent of collusion we observe. Indeed, in groups that communicated about relevant topics, $73 \%$ of outcomes in our first-price auctions and $86 \%$ of our second-price auctions culminated in a minimal price. In groups that did not have any meaningful discussions, none of the corresponding auctions, of either format, culminated in a price close to 0 . Since much of the relevant communication that took place revolved around an alternating strategy profile, we should expect efficiency levels to decrease for communicating groups: a simple alternation does not take into account the relative valuations of bidders in each round. Indeed, $75 \%$ of the outcomes in our first-price auctions in which meaningful communication took place were efficient, compared with $80 \%$ of the outcomes corresponding to groups with only irrelevant discussions. For second-price auctions, the difference between the two numbers is even more stark (and statistically significant): $57 \%$ of the auctions in which meaningful communication took place were efficient, compared with $87 \%$ of outcomes corresponding to irrelevant discussions.

To summarize, while a thorough investigation of repeated auctions would certainly be merited, the results from our auxiliary sessions suggest that: (1) repeated interaction does not allow more scope for collusion than does communication in one-shot settings; and (2) communication and repetition together do not allow more scope for collusion than do communication and transfers in one-shot settings. Furthermore, the success of collusive attempts in our repeated auctions is a double-edged sword for bidders. While it generates lower prices, it reduces efficiency.

\section{Conclusions and Discussion}

We report results from a sequence of experiments testing the impacts of communication and transfers on auction outcomes. The main message is that communication, especially when transfers are available, allows for a substantial amount of collusion in and of itself, even

\footnotetext{
${ }^{38}$ Some groups discuss both classes of strategies throughout the session.
} 
in one-shot settings. In fact, repetition, which has been one of the prevailing explanations for the emergence of collusion in the literature, does not appear to allow greater scope for collusion, even when combined with communication between rounds.

In the lab, the frequency of collusive outcomes is similar across auction formats, so neither is immune to collusive behavior. Furthermore, efficiency levels are not impacted by communication channels that allow for collusion in our one-shot setting. These observations are not fully in line with several basic theoretical predictions pertaining to such settings. Nonetheless, we see somewhat different patterns of misrepresentation during communication under the two formats that are consistent with strategic incentives suggested by theory.

In what follows, we discuss several behavioral models that relate to our results and some natural extensions of our basic experimental design.

\subsection{Norms of Transfers}

Recall our observations from Section 9: a successful collusion frequently led to a positive transfer, and conditional on a significant transfer being passed, transfers averaged at $44 \%$ and $45 \%$ of the winning bidder's surplus in our first- and second-price auctions, respectively. Furthermore, the modal fraction of the surplus transferred was $50 \%$ for both auction formats.

This may raise a suspicion that subjects operate under a norm that prescribes an equal division of the surplus (in the spirit of Andreoni and Bernheim, 2009). As it turns out, under both auction formats, were such a norm in place, the behavior patterns we observe in our data are consistent with equilibrium play.

Suppose players act according to the following protocol. In the communication stage, both reveal their value. Then, the low-value bidder submits a bid of 0 and the high-value bidder submits a bid of 0.01 (the smallest possible bid greater than 0 ). If both state the same value, both submit 0 . This protocol is incentive compatible under both auction formats. Indeed, both individuals have an incentive for the highest-value bidder to win the object at the lowest possible price since the surplus divided, and consequently their payoffs, are highest in that case. Furthermore, there is no incentive to out-bid at the bidding stage. This protocol echoes what we see in much of our data - subjects utilizing the communication phase to implement the reveal-collude strategy and then splitting the surplus of the winning bidder. ${ }^{39}$

This profile of actions is no longer an equilibrium if the prevailing norm was to split

\footnotetext{
${ }^{39}$ Notice that there could be a multiplicity of equilibria. In particular, in second-price auctions, winning bidders could submit arbitrary positive bids and we indeed see a substantial variance of high bids in our second-price auctions. These equilibria, which are equivalent with respect to outcomes, are welfare maximizing.
} 
the surplus unequally between the winning and losing bidders. Indeed, suppose the winning bidder was to keep a fraction $\alpha>1 / 2$ of the surplus and transfer a fraction $1-\alpha$. In this case, winning the object entails an advantage since a greater fraction of the surplus is then kept. This tilts individual incentives - they may prefer to win the object themselves even if it generates a slightly lower surplus. In fact, the above protocol does not constitute part of an equilibrium any longer. It can be shown that a bidder with a private value of $v$ would benefit from mis-reporting a value of $\frac{\alpha}{1-\alpha} v>v$ at the communication stage. In that respect, while norms of transfers are in line with much of our data, these conclusions are fragile to the precise norms in place.

\subsection{Other-regarding Preferences and Reciprocity}

One may worry that some of the generous transfers we observe in our experiments are an artifact of a form of other-regarding preferences that are often observed in laboratory settings (see, e.g., Fehr and Schmidt, 1999, Bolton and Ockenfels, 2000, and work that followed). Certainly, such other-regarding preferences cannot be overwhelmingly strong in our experimental setting. If they were, we would expect, for instance, that subjects submit zero bids in second-price auctions without communication in order to allow their opponent to gain the object at a low cost. Still, subjects may be putting some weight on their opponents' outcomes, in addition to their own. In their simplest form, models of other-regarding preferences pose utilities that are composed of two linear terms: one corresponding to one's own monetary outcomes and one corresponding to others' outcomes (this is the essence of the Fehr and Schmidt, 1999 model). Such a setup would imply corner solutions in our auctions - in treatments where transfers are available, agents should either transfer none of their surplus or all of it. This is clearly in contrast with what we observe in our data.

There are many ways to introduce non-linearities to the basic model that accounts for both one's own and others' outcomes. In order to illustrate the impacts of non-linearities, we consider a class of utilities as follows. The utility for bidder $i$ when her payoff is $\pi_{i}$ and her counter-part's payoff is $\pi_{j}$ is given by:

$$
U_{i}=\alpha \pi_{i}-(1-\alpha) f\left(\pi_{i}-\pi_{j}\right)
$$

where $\alpha \in[0,1]$ is a weight parameter that indicates how much bidder $i$ cares about her own payoff relative to the variation of payoffs within the pair. We assume that the inequality cost function $f$ is symmetric, $f(x)=f(-x)$, twice continuously differentiable, increasing in the distance between payoffs, $f^{\prime}(x) * \operatorname{sgn}(x)>0$, and convex, $f^{\prime \prime}(x)>0$ for all $x$. Now, consider the winner of the object in our treatment with transfers (regardless of the auction format), 
who has an object value of $v$ that she has gained for the price of $p$. If she makes a transfer of $t$, she receives a utility of:

$$
\alpha(v-p-t)-(1-\alpha) f(v-p-2 t)
$$

Maximization with respect to $t$ implies then that:

$$
v-p-2 t=\left(f^{\prime}\right)^{-1}\left(\frac{\alpha}{2(1-\alpha)}\right) \text {. }
$$

In other words, the net profits of the winning and the losing bidders should differ by a constant, the size of which depends on the weight put on one's own monetary outcomes relative to the egalitarian utility component. We stress that this solution does not depend on the auction format nor on what has transpired in the auction (namely, the ultimate price of the object). However, in our data, the difference between the surpluses of the two bidders exhibit a large variance and does not appear constant, even when conditioning on minimal price. ${ }^{40}$ Naturally, one could consider even more general functional forms, incomplete information on the weight $\alpha$ (in which case bids also serve as signals on the private parameter $\alpha$ ), etc. We leave such elaborations for future work. But simple models of other-regarding preferences, which are the common ones used in the literature, do not explain our observations.

Figure 8: Cumulative Distribution Functions of Individual Frequencies of Positive Transfers

Minimal Price

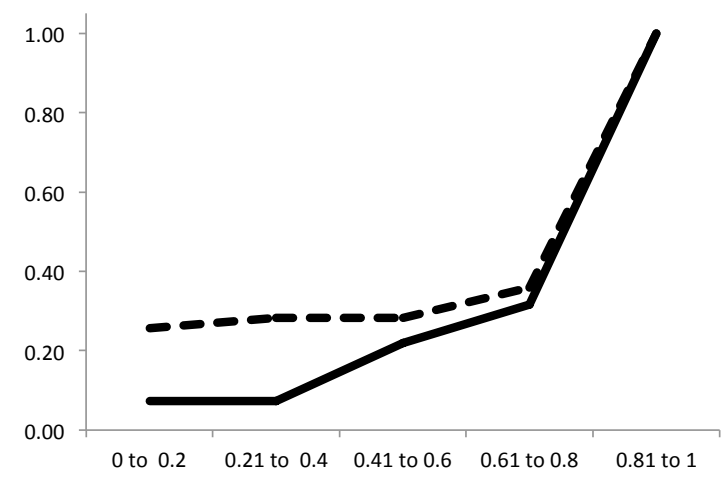

Above Minimal Price

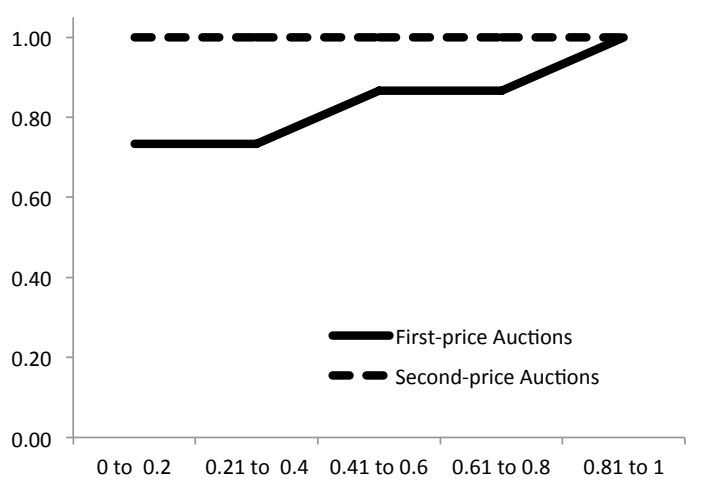

Our treatments allowing for transfers do point out to some reciprocal behavior. We

\footnotetext{
${ }^{40}$ Indeed, in our first-price auctions, the average difference between the surplus of the two bidders is 19 with a standard deviation of 25 and values varied between -10 and 92 . Similarly, in our second-price auctions the average difference is 14 with a standard deviation of 31 and values varied between -95 and 98 . Even when focusing only on auctions that resulted in the minimal price, the difference in surpluses varies significantly from auction to auction with standard deviations of similar size (26 in both auction formats).
} 
already saw in Section 9 that the pattern of transfers differed between auctions with a collusive outcome and those in which the ultimate price was not very low. In order to dig deeper into how individuals respond to final prices, we calculate for each individual subject the frequency of auctions in which he or she won the object and transferred a substantial amount (more than 2 points), when the auction culminated in the minimal price and when the auction culminated in a price higher than the minimal. Figure 8 depicts the corresponding cumulative distributions. The figure illustrates the strong response of subjects to the final price in the auction - subjects transfer substantial amounts at very high frequencies when prices are low. However, when prices are above the minimal price, the vast majority of subjects do not make transfers. This suggests that some reciprocity motives might be influencing subjects' behavior (see Fehr and Gachter, 2000 for an overview of some of the literature on reciprocity).

\subsection{Other Contract Structures}

In our experimental sessions, individuals could not commit to either actions or transfers. We believe the inspection of contracting structures and their impact on the fragility of different auction formats to collusion is a very interesting direction for future research. For example, one natural alternative contractual agreement would allow participants to commit to the transfers that would be made ex-ante. We ran six such additional sessions (three with firstprice auctions and three with second-price auctions, with 36 and 34 subjects, respectively). In these sessions, subjects observed their private values and were then able to communicate freely as in the Pure Communication Treatment. Once the communication was over, each bidder was asked to submit a transfer to the other bidder ( 0 or higher). These transfers were then announced simultaneously to the bidders. Following that, bidders submitted their bids. Ultimately, each bidder received their auction payment plus their net transfers (namely, the transfers made to them minus the transfers made by them). That is, subjects could commit to transfers that were paid ex-ante. ${ }^{41}$

In this auxiliary treatment, observations roughly mirror those we see in our transfers treatment without commitment. Indeed, with commitment to transfers subjects achieve similar levels of efficiency: $85 \%$ in our first-price and $95 \%$ in our second-price auctions. ${ }^{42}$ Further, revenues of the auctioneer (as measured by the average and median prices obtained

\footnotetext{
${ }^{41}$ This is reminiscent of the theoretical model in Eso and Schummer (2004). They consider second-price auctions in which bidders cannot communicate, but can commit to "bribes" prior to bidding. Unlike our setting, they assume that when a bribe is accepted, the receiver of the bribe is committed not to participate in the auction and illustrate the potential signaling power of bribes as well as the inefficiencies they may induce. See also the experiments reported in Llorente-Saguer and Zultan (2014).

${ }^{42}$ Robust standard errors are 0.02 and 0.03 , respectively, where errors are clustered by session.
} 
in these auctions) are significantly lower in the sessions with commitment to transfers compared with the ones without transfers and similar to the ones obtained by the auctioneer in the treatment with transfers and no commitment. ${ }^{43}$ These results are also mirrored by the frequencies of collusion. Collusion is detected in $59 \%$ of our first-price and $73 \%$ of our second-price auctions with transfers and commitment when collusion is defined relative to the equilibrium prices in the No Communication treatment (to be compared with the first column in Table 2). When collusion corresponds to subjects achieving the minimal price, we observe $44 \%$ collusive outcomes in our first-price auctions and $67 \%$ in our second-price auctions with transfers and commitment (to be compared with the second column in Table 2). Finally, when collusion is defined relative to the empirically estimated prices in the No Communication treatment, collusion rates are $74 \%$ and $89 \%$ in our first- and second-price auctions, respectively (to be compared with the third column in Table 2).

We note that in first-price auctions, collusion as well as the frequency of substantial transfers from winning bidders to losing bidders is somewhat lower in sessions with commitment to transfers than in sessions without commitment (in $47 \%$ of auctions with commitment winning bidders transfer points to losing bidders, compared with $65 \%$ in auctions without commitment). One potential reason is that commitment makes transfers risky as participants pay the transfers regardless of whether they ultimately receive the object or not. However, once we condition on the price being minimal, the difference between these two contract designs disappears, as the vast majority of winning bidders reward the losing bidders for achieving low prices (this happens in $84 \%$ of the auctions with commitment and in $79 \%$ of our auctions without commitment). In second-price auctions, the frequency of collusive outcomes and transfers are comparable between sessions with and without commitment. One potential interesting modification of this treatment would entail slightly more complex contracts in which transfers would be contingent on winning the object. This would mitigate the riskiness of transfers prior to the auction.

\subsection{Other Auction Formats}

Throughout the paper we focus on first- and second-price auctions. This seems like a natural first step, particularly given the prominence of these two auction formats in applications. It would be interesting to inspect the sensitivity of other auction formats to the availability of communication and transfers. For instance, we suspect that all-pay auctions would be rather fragile to communication since preference alignment arises more naturally through the structure of the auction itself: if bidders can successfully share their values, they can

\footnotetext{
${ }^{43}$ In first-price auctions, the average price is 22 and the median price is 10 , while in second-price auctions the corresponding numbers are 13 and 0 , respectively.
} 
both reduce the price experienced by the likely winner and avoid the participation cost of the prospective losing bidders of the auction. On the other hand, general $k$-price auctions (with $k \geq 3$ ) may exhibit different fragility to communication as coordination between more individuals is required to achieve successful collusion. The differences are subtle and are left as directions for future research.

\section{References}

[1] Abreu, Dilip, David Pearce, and Ennio Stachetti (1986), "Optimal Cartel Equilibria with Imperfect Monitoring," Journal of Economic Theory, 39, 251-269.

[2] Agranov, Marina and Chloe Tergiman (2014), "Communication in Multilateral Bargaining," Journal of Public Economics, forthcoming.

[3] Andreoni, James and B. Douglas Bernheim (2009), "Social Image and the 50-50 Norm: A Theoretical and Experimental Analysis of Audience Effects," Econometrica, 77(5), 1607-1636.

[4] Athey, Susan and Kyle Bagwell (2001), "Optimal Collusion with Private Information," RAND Journal of Economics, 32(3), 428-65.

[5] Blume, Andreas and Paul Heidhues (2004), "All Equilibria of the Vickrey Auction," Journal of Economic Theory, 114(1), 170-177.

[6] Baranski, Andrzej and John H. Kagel (2015), "Communication in Legislative Bargaining," Journal of the Economic Science Association, forthcoming.

[7] Berg, Joyce, John Dickhaut, and Kevin McCabe (1995), "Trust, Reciprocity, and Social History," Games and Economic Behavior, 10, 122-142.

[8] Bergemann, Dirk, Benjamin Brooks, and Stephen Morris (2013), "Extremal Information Structures in the First Price Auction," mimeo.

[9] Bolton, Gary E. and Axel Ockenfels (2000), "ERC: A Theory of Equity, Reciprocity, and Competition," The American Economic Review, 90(1), 166-193.

[10] Brandts, Jordi and David J. Cooper (2007), "It's What You Say, Not What You Pay: An Experimental Study of Manager-Employee Relationships in Overcoming Coordination Failure," Journal of the European Economic Association, 5, 1223-1268. 
[11] Brusco, Sandro and Giuseppe Lopomo (2002), "Collusion via Signalling in Simultaneous Ascending Bid Auctions with Multiple Objects and Complementarities," The Review of Economic Studies, 69(2), 407-436.

[12] Charness, Gary and Martin Dufwenberg (2006), "Promises and Partnership," Econometrica, 74, 1579-1601.

[13] Charness, Gary and Martin Dufwenberg (2011), "Participation," The American Economic Review, 101, 1213-1239.

[14] Charness, Gary, Imas, Alex and Uri Gneezy (2013), "Experimental methods: Eliciting risk preferences," Journal of Economics Behavior and Organization, 87, 43-51.

[15] Chwe, Michael S-Y. (1989), "The Discrete Bid First Auction," Economics Letters, 31, 303-306.

[16] Cox, James C., Bruce Roberson, and Vernon L. Smith (1982), "Theory and Behavior of Single Object Auctions," Research in Experimental Economics, 2, 1-43.

[17] Cox, James C., Vernon L. Smith, and James M. Walker (1983) "A Test that Discriminates Between Two Models of the Dutch-First Auction Non-Isomorphism," Journal of Economics Behavior and Organization, 14, 205-219.

[18] Crawford, Vincent (1998), "A Survey of Experiments on Communication via Cheap Talk," Journal of Economic Theory, 78, 286-298.

[19] Dyer, Douglas, John H. Kagel, and Dan Levin (1989), "A Comparison of Naive and Experienced Bidders in Common Value Offer Auctions: A Laboratory Analysis," The Economic Journal, 99(394), 108-115.

[20] Eso, Peter and James Schummer (2004), "Bribing and Signaling in Second Price Auctions," Games and Economic Behavior, 47(2), 299-324.

[21] Fehr, Ernst and Simon Gachter (2000), "Fairness and Retaliation: The Economics of Reciprocity," The Journal of Economic Perspectives, 14(3), 159-181.

[22] Fehr, Ernst and Klaus M. Schmidt (1999), "A Theory of Fairness, Competition, and Cooperation," The Quarterly Journal of Economics, 114(3), 817-868.

[23] Fudenberg, Drew and Jean Tirole (1991), Game Theory, The MIT Press.

[24] Gerardi, Dino (2004), "Unmediated Communication in Games with Complete and Incomplete Information," Journal of Economic Theory, 114, 104-131. 
[25] Gneezy, Uri and Jan Potters (1997), "An experiment on risk taking and evaluation periods," The Quarterly Journal of Economics, 112(2), 631-645.

[26] Goeree, Jacob K. and Leeat Yariv (2011), "An Experimental Study of Collective Deliberation," Econometrica, 79(3), 893-921.

[27] Grether, David, David Porter, and Matthew Shum (2014), "Cyber-shilling in Automobile Auctions: Evidence from a Field Experiment," American Economic Journal: Microeconomics, forthcoming.

[28] Harstad, Ronald J. (1991), "Asymmetric Bidding in Second-price, Common-value Auctions," Economics Letters, 35, 249-252.

[29] Hendricks, Kenneth and Robert H. Porter (1989), "Collusion in Auctions," Annales d'Économie et de Statistique, 15/16, 217-230.

[30] Hong, Han and Matthew Shum (2002), "Increasing Competition and the Winner's Curse: Evidence from Procurement," The Review of Economic Studies, 69, 871-898.

[31] Isaac, R. Mark and James M. Walker (1985). "Information and Conspiracy in Sealed Bid Auctions," Journal of Economic Behavior and Economic Organization, 6, 139-159.

[32] Kagel, John H. (1995), "Auctions: a survey of experimental research," in The Handbook of Experimental Economics, Alvin E. Roth and John H. Kagel, Editors, Princeton University Press.

[33] Kagel, John H. and Dan Levin (1993), "Independent Private Value Auctions: Bidder Behaviour in First-, Second- and Third-Price Auctions with Varying Numbers of Bidders," The Economic Journal, 103(419), 868-879.

[34] Kagel, John H. and Dan Levin (2011), "Auctions: A Survey of Experimental Research, 1995 - 2010," forthcoming in The Handbook of Experimental Economics, Volume 2.

[35] Kagel, John H. and Alvin E. Roth (1997), The Handbook of Experimental Economics, Princeton University Press.

[36] Krishna, Vijay (2002), Auction Theory, Academic Press.

[37] Kwasnica, Anthony M. (2002), "A Theory of Collusion in Multiple Object Simultaneous Auctions," mimeo.

[38] Lebrun, Bernard (2004), "Uniqueness of Equilibrium in First-Price Auctions," mimeo. 
[39] Llorente-Saguer, Aniol and Ro’i Zultan (2014), "Auction Mechanisms and Bidder Collusion: Bribes, Signals and Selection," mimeo.

[40] Lopomo, Giuseppe, Leslie M. Marx, and Peng Sun (2011), "Linear Programming for Mechanism Design: An Application to Bidder Collusion in First Price Auctions," Review of Economic Design, 15, 177-211.

[41] Marshall, Robert C. and Leslie M. Marx (2012), The Economics of Collusion: Cartels and Bidding Rings, The MIT Press.

[42] Maskin, Eric and John Riley (2003), "Uniqueness of Equilibrium in Sealed High-bid Auctions," Games and Economic Behavior, 45(2), 395-409.

[43] McAfee, R. Preston and John McMillan (1992), "Bidding Rings," The American Economic Review, 82(3), 579-599.

[44] Oprea, Ryan, Gary Charness, and Dan Friedman (2015), "Continuous Time and Communication in a Public-goods Experiment," Journal of Economic Behavior and Organization, forthcoming.

[45] Pesendorfer, Martin (2000), "A Study of Collusion in First-price Auctions," The Review of Economic Studies, 67, 381-411.

[46] Skrzypacz, Andrzej and Hugo Hopenhayn (2004), "Tacit Collusion in Repeated Auctions," Journal of Economic Theory, 114, 153-169. 